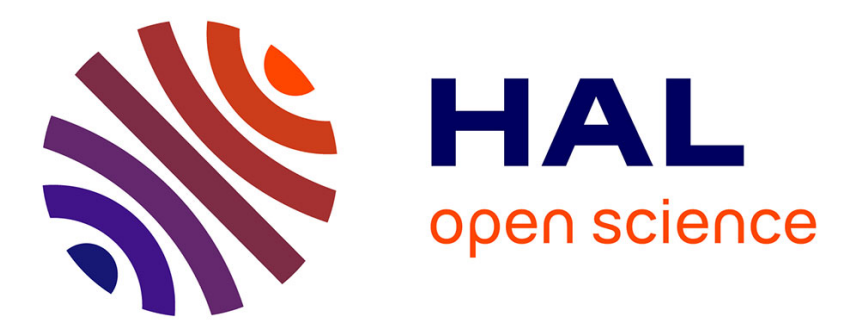

\title{
Vibration of a Timoshenko beam supporting arbitrary large pre-deformation
}

Loïc Le Marrec, Jean Lerbet, Lalaonirina Rakotomanana-Ravelonarivo

\section{To cite this version:}

Loïc Le Marrec, Jean Lerbet, Lalaonirina Rakotomanana-Ravelonarivo. Vibration of a Timoshenko beam supporting arbitrary large pre-deformation. Acta Mechanica, 2018, 229 (1), pp.109-132. 10.1007/s00707-017-1953-x . hal-01599177

\section{HAL Id: hal-01599177 \\ https://hal.science/hal-01599177}

Submitted on 6 Jan 2020

HAL is a multi-disciplinary open access archive for the deposit and dissemination of scientific research documents, whether they are published or not. The documents may come from teaching and research institutions in France or abroad, or from public or private research centers.
L'archive ouverte pluridisciplinaire HAL, est destinée au dépôt et à la diffusion de documents scientifiques de niveau recherche, publiés ou non, émanant des établissements d'enseignement et de recherche français ou étrangers, des laboratoires publics ou privés. 
Loïc Le Marrec $\mathbb{D}$ · Jean Lerbet ·

Lalaonirina R. Rakotomanana

\title{
Vibration of a Timoshenko beam supporting arbitrary large pre-deformation
}

\begin{abstract}
We present an induced geometrically exact theory for the three-dimensional vibration of a beam undergoing finite transformation. The beam model coincides with a curvilinear Cosserat body and may be seen as an extension of the Timoshenko beam model. No particular hypothesis is used for the constitutive laws (in the framework of hyperelasticity), the geometry at rest or boundary conditions. The method leads to a weak formulation of the equations of vibration. The obtained internal energy is symmetric and leads to a self-adjoint operator that casts into a geometrical and material parts. Both may be written explicitly in terms of the finite transformation. The results are applied on the vibration of a beam supporting a finite longitudinal strain. The nonlinear effects according to the pre-stress are explicitly detailed for this example: instability, buckling.
\end{abstract}

\section{Introduction}

Engineering applications of vibration, such as structural health monitoring and more widely nondestructive testing, are well developed and documented. In the high frequency domain (i.e., for small wavelengths compared to the structure size) the problem is mainly associated to ultrasonic propagation. In the large frequency domain, the vibrations are strongly affected by the effective properties of the structure and its boundary conditions [1,2]. Of course, a wide literature exists on the sensitivity of the dynamical response to geometry [3], constitutive laws [4-8] and boundary conditions [9]. However, the theoretical investigation of the effect of a finite transformation has not attracted so much attention.

The well-known exception is the string for which the relation between tension (pre-stress) and frequencies is stated by Marin Mersenne in his 1637 work. An instructive bibliography can be found in the conclusion of [10]. However, in addition to the string, the problem of finite transformation is observed in many structural configurations. The present paper focuses on beams and then possible applications range from the pillar bearing a heavy swiveling deck to the tension in micro-ships under a thermal dilation of its support and includes guy ropes.

The classical Euler-Bernoulli beam model is suitable for most engineering applications [11]. However, it is rather limited to relatively low frequencies and thin beams. The Timoshenko beam model enlarges the frequency domain of validity and allows applications for relatively thicker beams, e.g., [12-14]. The timoshenko model

L. Le Marrec $(\varangle) \cdot$ L. R. Rakotomanana

IRMAR, UMR CNRS 6625, Université de Rennes 1, Campus Beaulieu, 35042 Rennes, France

E-mail: loic.lemarrec@univ-rennes1.fr

L. R. Rakotomanana

E-mail: lalaonirina.rakotomanana-ravelonarivo@univ-rennes1.fr

J. Lerbet

IBISC, Univ Evry, Université Paris-Saclay, 91025 Evry, France

E-mail: jlerbet@gmail.com 
introduces transversal shear and does not neglect the rotational inertia of the section. Introducing new degrees of freedom, it describes two types of standing waves for a given frequency $[15,16]$. The kinematics of a threedimensional beam allowing torsion and extension refer to the analysis of a curvilinear Cosserat medium [17] and constitutes the general framework of the paper.

Theoretical (e.g., $[18,19])$ and numerical (e.g., [20]) approaches may be performed to tackle the effect of pre-stress on the superimposed waves. The problem of finite displacement may be found for a large variety of application, too: from wind turbine [21] to musical instrument [22]. In general, three difficulties are present: the nonlinear stress-strain relation (large strain and nonlinear constitutive laws of material), the moving frame induced by large displacement, and the load sensitivity to the transformation (leading to the distinction between dead and followers loads). This explains why, in most of the cases, the methods are dedicated to a particular application that permits simplifications and in some case an analytical formulation [19,23].

Indeed, such analysis requires to extend the theoretical modeling of finite transformation of a beam developed by [24] and generalized in [25]. In the static regime, Bernoulli-Euler [26] and Timoshenko-type kinematic formulations [27] were used in the plane case in the framework of the Reissner approach [28]. The dynamical problem for a shearing rod may be found in [29,30]. Later, in [31], a the full coordinate-free approach of the dynamics of curvilinear systems was developed. It involves calculations on Lie groups for generating the dynamic equations of a similar model as the one of the present paper. However, it did not make a bridge with the usual concepts of continuum and structural mechanics especially regarding the strain analysis. Moreover, it did not tackle any modal analysis, too.

From our knowledge, a general formulation of the vibrations superimposed on a finite transformation is not addressed in these previous theoretical works. Nowadays, the dynamic response of a beam supporting finite strain is essentially numerically oriented [32]. More precisely, the problem of infinitesimal vibrations superimposed on the finite transformation is based on the determination of the continuum tangent operator. Following, the strategy of [24], we extend the work in order to give a methodology allowing us to write the weak and strong formulation of the vibration equation of a beam of general material around an arbitrary finite transformation.

Some basics on the finite transformation of a three-dimensional body are presented first. Particular attention must be paid to avoid some hypothesis on the material properties and the corresponding constitutive laws. The second section aims to introduce the beam model in the sense of a Cosserat one-dimensional element moving in the three-dimensional world. In that sense, the whole paper gives a special place to the directors as preferred basis of tensorial objects. The consequences on the kinematics and dynamics are presented without additional hypothesis. The weak formulation of the dynamical equation is then obtained by careful integration over the cross section. This gives the opportunity to introduce the corotational derivative of strains (also known as Jaumann derivative). In the fifth section, the general problem of superimposed small perturbation (waves) is presented as a (Gâteaux) linearization of the additional perturbations around the finite transformation. The general methodology to obtain weak and strong formulation of the (superimposed) wave equation is given without further hypothesis. For inertia and internal power, the question of the self-adjoint character of the mass and rigidity operators are posed, whereas the effects of the load (follower or dead) are studied for the external virtual load. In the last section, an example is proposed in order to show how analytical and explicit equation may be recovered. Even if this example is very simple (dead longitudinal pre-stress of a straight beam made of a Kirchhoff-Saint Venant material), the three-dimensional equations (torsion, flexion, and extension) of superimposed vibrations show a large number of effects that are briefly exposed and discussed. Confrontation with other models as string and ropes is performed and shows the large versatility of the presented approach.

\section{General considerations for finite transformations of a bounded body}

We consider a bounded material body $\mathcal{B}$. Its reference configuration $\boldsymbol{\Phi}_{0}$ is defined as the configuration where the body is at rest (stress-free) in absence of external load. Let $\partial \mathcal{B}$ be the surface boundary of $\mathcal{B}$ where traction f is eventually prescribed (per unit initial area of the reference configuration). The body is subjected to a body force field $\rho_{0} \mathbf{b}$, where $\rho_{0}$ is the mass density in the reference configuration (see Fig. 1).

\subsection{Kinematics}

We denote a fixed arbitrary origin $O$ and $M$ a point of the body placed at $\mathbf{O M}=X_{I} \mathbf{D}_{\mathbf{I}}$ in the reference configuration. After finite transformation, the point $M$ is localized at $\mathbf{O M}=x_{i} \mathbf{d}_{i}$. The local bases $\left\{\mathbf{D}_{\mathbf{I}}\right\}$ and 

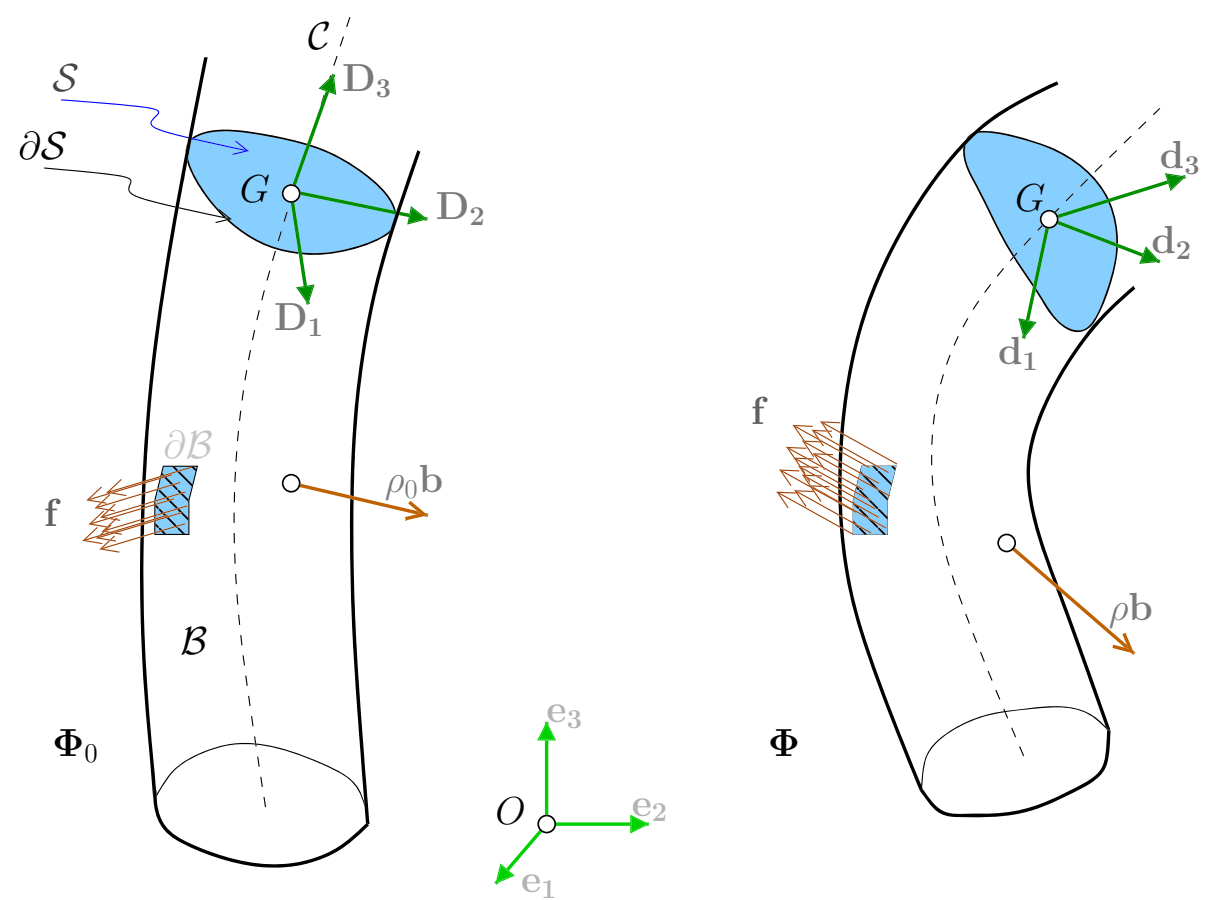

Fig. 1 Reference configuration $\boldsymbol{\Phi}_{0}$ (left) and current configuration $\boldsymbol{\Phi}$. A priori the force densities may change along the transformation (followers load for example). The cross section is normal to the centerline $\mathcal{C}$ in the reference configuration but not necessarily in the current configuration: $\mathbf{d}_{\mathbf{3}}$ is not tangent to $\mathcal{C}$

$\left\{\mathbf{d}_{\mathbf{i}}\right\}$ are associated with the reference and current configuration, respectively, and may be distinct from the Cartesian basis $\left\{\mathbf{e}_{\mathbf{I}}\right\}$.

The deformation gradient is $\mathbf{F}:=\nabla_{\mathbf{X}} \mathbf{O M}$ and reads in terms of components $\mathbf{F}=F_{I}^{i} \mathbf{d}_{\mathbf{i}} \otimes \mathbf{D}_{\mathbf{I}}$. However, all along the paper $\left\{\mathbf{D}_{\mathbf{I}}\right\}$ and $\left\{\mathbf{d}_{\mathbf{i}}\right\}$ are both orthonormal bases, and the low or high position of tensor's indices is not meaningful and all indices will be lowered in the following.

For finite transformation, the strain may be described by the Green-Lagrange strain tensor:

$$
\mathbf{E}:=\frac{1}{2}\left(\mathbf{F}^{T} \mathbf{F}-\mathbf{I}\right), \quad \text { then } \mathbf{E}=\frac{1}{2}\left(F_{i I} F_{i J}-\delta_{I J}\right) \mathbf{D}_{\mathbf{I}} \otimes \mathbf{D}_{\mathbf{J}} .
$$

\subsection{Stress}

The second Piola-Kirchhoff stress tensor $\mathbf{S}$ is associated with the Green-Lagrange strain. From a given configuration of the three-dimensional body, the local stress increment $\delta \mathbf{S}$ is related to the increment of local strain $\delta \mathbf{E}$ by a relation of the form:

$$
\delta \mathbf{S}=\mathrm{C} \delta \mathbf{E}, \quad \text { or explicitly } \delta S_{I J}=\mathrm{C}_{I J K L} \delta E_{K L} .
$$

Here stress and strain are described by purely Lagrangian tensors. $C$ is an elasticity tensor (with a priori dependence on $\mathbf{E}$ ). The angular momentum balance induces left minor symmetry $\mathrm{C}_{I J K L}=\mathrm{C}_{J I K L}$, whereas the symmetry of the strain tensor induces right minor symmetry $\mathrm{C}_{I J K L}=\mathrm{C}_{I J L K}$ [33]. Major symmetry is ensured in the case of a hyperelastic material. If the stress-strain relation is continuous around the reference configuration:

$$
\mathrm{C}=\frac{\partial \mathbf{S}}{\partial \mathbf{E}} \quad \text { or } \quad \mathrm{C}_{I J K L}=\frac{\partial S_{I J}}{\partial E_{K L}} .
$$

This relation is general and may be used even for a class of material for which $C$ depends on the strain: $C(\mathbf{E})$.

The nominal or first Piola-Kirchhoff stress tensor $\mathbf{P}=P_{i J} \mathbf{d}_{\mathbf{i}} \otimes \mathbf{D}_{\mathbf{J}}$ is related to $\mathbf{S}$ by

$$
\mathbf{P}=\mathbf{F S}, \quad \text { then } \quad P_{i J}=F_{i K} S_{K J} .
$$

Symmetry of $\mathbf{S}$ is imposed by the law of angular momentum and induces: $\mathbf{P F}^{T}=\mathbf{F} \mathbf{P}^{T}$. 


\subsection{Introduction to weak formulation}

We recall some basic points of the variational formulation for the dynamics of an elastic body. The principle of virtual power takes the following form, e.g., [34]

$$
\int_{\mathcal{B}} \rho_{0} \mathbf{b} \cdot \delta \mathbf{u} \mathrm{d} V+\int_{\partial \mathcal{B}} \mathbf{f} \cdot \delta \mathbf{u} \mathrm{d} A=\int_{\mathcal{B}} \rho_{0} \frac{\partial^{2} \mathbf{u}}{\partial t^{2}} \cdot \delta \mathbf{u} \mathrm{d} V+\int_{\mathcal{B}} \mathbf{P}: \delta \mathbf{F} \mathrm{d} V, \quad \forall \delta \mathbf{u} \in W
$$

where $\mathbf{u}$ is the displacement of any point of the three-dimensional body and $W$ is the space of kinematically compatible virtual displacement, usually called the space of variation for the continuum. The various terms in the preceding equation are the virtual power of inertia, the virtual power of external loading and internal virtual power, respectively:

$$
\begin{aligned}
& \mathcal{P}_{j}(\Phi, \delta \Phi)=\int_{\mathcal{B}} \rho_{0} \frac{\partial^{2} \mathbf{u}}{\partial t^{2}} \cdot \delta \mathbf{u} \mathrm{d} V, \\
& \mathcal{P}_{e}(\Phi, \delta \Phi)=\int_{\mathcal{B}} \rho_{0} \mathbf{b} \cdot \delta \mathbf{u} \mathrm{d} V+\int_{\partial \mathcal{B}} \mathbf{f} \cdot \delta \mathbf{u} \mathrm{d} A, \\
& \mathcal{P}_{i}(\Phi, \delta \Phi)=\int_{\mathcal{B}} \mathbf{P}: \delta \mathbf{F} \mathrm{d} V .
\end{aligned}
$$

At this stage, an important point must be clarified: all these terms are calculated in the reference configuration. Hence, they are computed by an integration along the body in the reference configuration. This explains the choice of the mass density $\rho_{0}$ and of the capital letters for volume and area density ( $\mathrm{d} V$ and $\mathrm{d} A$, respectively).

Another formulation of the internal virtual power may be obtained according to the dual tensor $\mathbf{S}$ and $\mathbf{E}$. Indeed, remembering the relation $\mathbf{P}=\mathbf{F S}$, we have for the internal power density: $\mathbf{P}: \delta \mathbf{F}=\mathbf{S}: \mathbf{F}^{T} \delta \mathbf{F}$. Observing that $\delta \mathbf{E}=(1 / 2)\left((\delta \mathbf{F})^{T} \mathbf{F}+\mathbf{F}^{T} \delta \mathbf{F}\right)$ and symmetry of $\mathbf{S}$, we obtain (without any hypotheses on the material behavior):

$$
\mathbf{P}: \delta \mathbf{F}=\mathbf{S}: \delta \mathbf{E}=S_{I J} \delta E_{I J},
$$

with $\delta E_{I J}=(1 / 2)\left(\delta F_{k I} F_{k J}+F_{k I} \delta F_{k J}\right)$.

\section{Beam model as Cosserat continuum}

For a body having a large slenderness ratio, a beam approximation is used in the general context of a Cosserat continuum.

\subsection{Kinematics}

We consider a Cosserat beam model [24] defined by a spatial material curve $\mathcal{C}$. Practically the curve corresponds to the positions of the section mass center $G$. At each time $t$, this latter is defined by $(S, t) \in \mathbb{R}^{2} \longrightarrow$ $\mathbf{O G}(S, t)=\varphi(S, t)$, where $S$ is a material curvilinear coordinate of $\mathcal{C}$. The section of the beam is supposed to be plane and rigid. Each point of this curve is associated with a rigid orthonormal base of directors $\left\{\mathbf{d}_{i}\right\}$. These directors are time-dependent $\mathbf{d}_{\mathbf{i}}(S, t)$ with $\mathbf{d}_{\mathbf{i}}(S, 0)=\mathbf{D}_{\mathbf{i}}(S)$. The orientation of any section $\mathcal{S}$ is defined by $(S, t) \in \mathbb{R}^{2} \longrightarrow \mathbf{d}_{\alpha}(S, t), \alpha=1,2$ where $\mathbf{d}_{\alpha}$ belongs to the section plane. This leads to the definition of $\mathbf{d}_{3}:=\mathbf{d}_{1} \times \mathbf{d}_{2}$ that is normal to the section.

Therefore, the position of any material point $M$ of the beam in the deformed configuration is defined by the mapping, e.g., [35] (with summation over 1 and 2 for repeated Greek indices and from 1 to 3 for repeated Latin indices):

$$
\left(\xi_{1}, \xi_{2}, S, t\right) \in \mathbb{R}^{3} \times \mathbb{R} \longrightarrow \mathbf{O M}\left(\xi_{1}, \xi_{2}, S, t\right)=\boldsymbol{\varphi}(S, t)+\xi_{\alpha} \mathbf{d}_{\alpha}(S, t) \in \mathbb{R}^{3}
$$

Note that $\xi_{1}, \xi_{2}$ are time invariant in conformity with rigid section hypotheses.

A priori the transversal shear is not neglected, and the normal vector of the section $\mathbf{d}_{\mathbf{3}}$ is not tangent to the material curve $\mathcal{C}$. The plane $\left(\mathbf{d}_{1}, \mathbf{d}_{2}\right)$ is a material plane whereas the vector $\mathbf{d}_{3}$ is not material fiber. Because $\mathbf{d}_{3}$ is always a unit vector, it is not convected by the transformation. Hence $\left\{\mathbf{d}_{\mathbf{i}}\right\}$ is neither a material nor a purely convected frame, but a specific spatial frame associated with the current configuration. 
By construction, a rotation tensor $\mathbf{Q}(S, t)$ relates, at any time, each director to the reference one: $\mathbf{d}_{\mathbf{i}}(S, t)=$ $\mathbf{Q}(S, t) \mathbf{D}_{\mathbf{i}}(S)$ then $\mathbf{D}_{\mathbf{i}}(S)=\mathbf{Q}^{T}(S, t) \mathbf{d}_{\mathbf{i}}(S, t)$ or equivalently $\mathbf{Q}=\mathbf{d}_{\mathbf{i}} \otimes \mathbf{D}_{\mathbf{i}}$. On the other hand, the reference director of any section is related to the Cartesian basis by a rotation $\mathbf{D}_{\mathbf{i}}(S)=\mathbf{R}(S) \mathbf{e}_{\mathbf{i}}$ where $\mathbf{R}(S)$ is intrinsically defined by the curve $\mathcal{C}$ of the beam at rest.

Spatial and temporal derivation of the directors involve twist $\boldsymbol{\kappa}$ and spin vector $\boldsymbol{\omega}$, respectively. Indeed, for spatial derivation:

$$
\frac{\partial \mathbf{d}_{\mathbf{i}}}{\partial S}=\frac{\partial \mathbf{Q}}{\partial S} \mathbf{Q}^{\mathrm{T}} \mathbf{d}_{\mathbf{i}}+\mathbf{Q} \frac{\partial \mathbf{R}}{\partial S} \mathbf{R}^{\mathrm{T}} \mathbf{Q}^{\mathrm{T}} \mathbf{d}_{\mathbf{i}}
$$

We introduce twist $\boldsymbol{\kappa}$ as the axial vector associated with the skew-symmetric tensor $\mathbf{K}$ :

$$
\mathbf{K}:=\frac{\partial \mathbf{Q}}{\partial S} \mathbf{Q}^{\mathrm{T}}+\mathbf{Q} \frac{\partial \mathbf{R}}{\partial S} \mathbf{R}^{\mathrm{T}} \mathbf{Q}^{\mathrm{T}}, \quad \boldsymbol{\kappa}(S, t):=\operatorname{vec}(\mathbf{K}) .
$$

The same methodology is performed for time derivation:

$$
\frac{\partial \mathbf{d}_{\mathbf{i}}}{\partial t}=\frac{\partial \mathbf{Q}}{\partial t} \mathbf{Q}^{\mathrm{T}} \mathbf{d}_{\mathbf{i}},
$$

and spin $\omega$ is the axial vector associated with the skew-symmetric tensor $\mathbf{W}$ :

$$
\mathbf{W}:=\frac{\partial \mathbf{Q}}{\partial t} \mathbf{Q}^{\mathrm{T}}, \quad \omega(S, t):=\operatorname{vec}(\mathbf{W}) .
$$

Note that $\mathbf{K}$ and $\mathbf{W}$ are updated Lagrangian-like tensors in the sense that they are associated with directors in the current configuration. They are parametrized by the reference arc length $S$. The spin and twist may be naturally expressed in this specific basis: $\boldsymbol{\omega}(S, t)=\omega_{i} \mathbf{d}_{\mathbf{i}}$ and $\boldsymbol{\kappa}(S, t)=\kappa_{i} \mathbf{d}_{\mathbf{i}}$.

We have finally the fundamental kinematical relation:

$$
\frac{\partial \mathbf{d}_{\mathbf{i}}}{\partial S}=\boldsymbol{\kappa} \times \mathbf{d}_{\mathbf{i}}, \quad \frac{\partial \mathbf{d}_{\mathbf{i}}}{\partial t}=\boldsymbol{\omega} \times \mathbf{d}_{\mathbf{i}} .
$$

It is the occasion to introduce a practical remark:

$$
\frac{\partial \boldsymbol{\kappa}}{\partial t}-\omega \times \boldsymbol{\kappa}=\frac{\partial \omega}{\partial S},
$$

that is related to the Maurer-Cartan form [36].

\subsection{Deformation}

From the beam motion defined by the map Eq. (10), the deformation gradient $\mathbf{F}:=\nabla_{\mathbf{X}} \mathbf{O M}$ is given by [35]:

$$
\mathbf{F}=F_{i J} \mathbf{d}_{\mathbf{i}} \otimes \mathbf{D}_{\mathbf{J}}=\frac{\partial \mathbf{O M}}{\partial \xi_{\alpha}} \otimes \mathbf{D}_{\alpha}+\frac{\partial \mathbf{O M}}{\partial S} \otimes \mathbf{D}_{3} .
$$

Using Eqs. (10) and (11), we have for $\mathbf{G M}=\xi_{\alpha} \mathbf{d}_{\alpha}$ a simple derivation for the rigid section:

$$
\frac{\partial \mathbf{G M}}{\partial S}=\boldsymbol{\kappa} \times \mathbf{G M}
$$

and then

$$
\mathbf{F}=\mathbf{d}_{\alpha} \otimes \mathbf{D}_{\alpha}+\left(\frac{\partial \varphi}{\partial S}+\kappa \times \mathbf{G M}\right) \otimes \mathbf{D}_{3}
$$

Introducing the strain vector of the beam, e.g., [24]:

$$
\boldsymbol{\varepsilon}(S, t):=\frac{\partial \boldsymbol{\varphi}}{\partial S}(S, t)-\mathbf{d}_{3}(S, t),
$$


the deformation gradient can be write as $\mathbf{F}=\mathbf{Q}(\mathbf{I}+\mathbf{H})$ where $\mathbf{Q}$ is the rotation of the section, $\mathbf{I}$ is the identity tensor, and $\mathbf{H}=\left(\mathbf{d}_{\mathbf{i}} \cdot(\boldsymbol{\varepsilon}+\boldsymbol{\kappa} \times \mathbf{G M})\right) \mathbf{D}_{\mathbf{i}} \otimes \mathbf{D}_{\mathbf{3}}$. Up to a rotation, the deformation gradient is entirely defined by the knowledge of $\mathbf{H}$ (and then by $\boldsymbol{\varepsilon}$ and $\boldsymbol{\kappa}$ ).

On the other hand, the Green-Lagrange strain tensor is:

$$
\mathbf{E}=\frac{1}{2}\left(\mathbf{F}^{T} \mathbf{F}-\mathbf{I}\right)=\frac{1}{2}\left(\mathbf{H}^{T}+\mathbf{H}+\mathbf{H}^{T} \mathbf{H}\right)
$$

Again $\varepsilon$ and $\boldsymbol{\kappa}$ govern the strain even for finite transformation. Using these quantities, the Green-Lagrange strain $\mathbf{E}=\frac{1}{2}\left(F_{i I} F_{i J}-\delta_{I J}\right) \mathbf{D}_{\mathbf{I}} \otimes \mathbf{D}_{\mathbf{J}}$ takes the form of an anti-plane tensor [37]:

$$
\begin{aligned}
\mathbf{E}= & \left(\mathbf{d}_{\alpha} \cdot(\boldsymbol{\varepsilon}+\boldsymbol{\kappa} \times \mathbf{G M})\right) \frac{\mathbf{D}_{\alpha} \otimes \mathbf{D}_{3}+\mathbf{D}_{3} \otimes \mathbf{D}_{\alpha}}{2} \\
& +\ldots\left(\left(\mathbf{d}_{\mathbf{3}} \cdot(\boldsymbol{\varepsilon}+\boldsymbol{\kappa} \times \mathbf{G M})\right)+\frac{1}{2}\|\boldsymbol{\varepsilon}+\boldsymbol{\kappa} \times \mathbf{G M}\|^{2}\right) \mathbf{D}_{3} \otimes \mathbf{D}_{3}
\end{aligned}
$$

where $\boldsymbol{\varepsilon}+\boldsymbol{\kappa} \times \mathbf{G M}=\frac{\partial \mathbf{O M}}{\partial S}-\mathbf{d}_{\mathbf{3}}$, then $E_{33}=\frac{1}{2}\left(\left\|\frac{\partial \mathbf{O M}}{\partial S}\right\|^{2}-1\right)$

It must be pointed out that $\mathbf{E}$ is a Lagrangian tensor whatever the use of the components of spatial vectors $\varepsilon$ and $\boldsymbol{\kappa}$. The interest of using parametrization of these two vectors by the reference arc length is now clarified: The geometrical parametrization of the Green-Lagrange strain tensor is purely related to coordinates in the reference configuration: $\left(\xi_{1}, \xi_{2}, S\right)$.

\subsection{Forces and moment}

In addition to kinematics, beam dynamics requires the definition of associated internal forces and moments. The resultant force $\mathrm{N}$ (or internal resultant stress) acting on the section $\mathcal{S}$ and the moment M per unit reference length in an arbitrary section $\mathcal{S}$, are easily obtained by means of the nominal Piola-Kirchhoff stress tensor (Eq. 4):

$$
\mathrm{N}:=\int_{\mathcal{S}} \mathbf{P}\left(\mathbf{D}_{3}\right) \mathrm{d} A=N_{i} \mathbf{d}_{\mathbf{i}}, \quad \mathbf{M}:=\int_{\mathcal{S}} \mathbf{G M} \times \mathbf{P}\left(\mathbf{D}_{3}\right) \mathrm{d} A=M_{i} \mathbf{d}_{\mathbf{i}}
$$

where $\mathrm{d} A$ is the area element of the section (unchanged by the transformation according to the rigid section hypothesis). $\mathrm{N}$ and $\mathrm{M}$ take values on the reference configuration, and their components are naturally given relative to the current basis $\left\{\mathbf{d}_{\mathbf{i}}\right\}$.

\section{Variational approach}

We present the variational approach for finite transformation of the beam.

\subsection{Admissible variation for the beam model}

Since the variational approach is expanded all along the paper, a brief review on the admissible variations of the kinematics measures have to be first defined clearly.

As previously mentioned, a configuration of the beam is specified by the position of the center line and the orientation of the director frame

$$
\Phi(S):=(\varphi(S), \mathbf{Q}(S)) \in \mathbb{R}^{3} \times \mathcal{S O}(3)
$$

where $\mathcal{S O}(3):=\left\{\mathbf{Q}: \mathbb{R}^{3} \rightarrow \mathbb{R}^{3}\right.$ linear $\mid \mathbf{Q}^{T} \mathbf{Q}=\mathbf{I}$ and $\left.\operatorname{Det} \mathbf{Q}=1\right\}$ is the special orthogonal group. Let $\delta \boldsymbol{\varphi}$ be an infinitesimal displacement of the center line superimposed on the center line $\boldsymbol{\varphi}$ and let $\tilde{\mathbf{Q}}$ a skew-symmetric tensor representing an infinitesimal rotation superimposed on an arbitrary rotation $\mathbf{Q}$ (here the dependence $(S, t)$ is implicit). Hence the perturbated configuration $\Phi_{\varepsilon}:=\left(\boldsymbol{\varphi}_{\varepsilon}, \mathbf{Q}_{\varepsilon}\right) \in \mathbb{R}^{3} \times \mathcal{S O}(3)$ is obtained by:

$$
\boldsymbol{\varphi}_{\varepsilon}=\boldsymbol{\varphi}+\delta \boldsymbol{\varphi}, \quad \mathbf{Q}_{\varepsilon}=\exp [\tilde{\mathbf{Q}}] \mathbf{Q} .
$$


By exponentiation of a skew-symmetric tensor, one obtains an orthogonal tensor. Thus, $\mathbf{Q}_{\varepsilon}$ is an orthogonal tensor. $\tilde{\mathbf{Q}}$ is an element of $\operatorname{so}(3):=\left\{\mathbf{Q}: \mathbb{R}^{3} \rightarrow \mathbb{R}^{3}\right.$ linear $\left.\mid \mathbf{Q}+\mathbf{Q}^{T}=\mathbf{0}\right\}$ which is the Lie algebra of all skew-symmetric tensors. Due to the isomorphism between $s o(3)$ and $\mathbb{R}^{3}, \tilde{\mathbf{Q}}$ can be replaced by its associated axial vector $\delta \omega$ such that

$$
\tilde{\mathbf{Q}} \boldsymbol{v}=\delta \omega \times \boldsymbol{v} \quad \forall \boldsymbol{v} \in \mathbb{R}^{3}
$$

and denoting a superimposed infinitesimal rotation. Indeed $\delta \omega$ is a kinematically admissible virtual variation of the directors' rotation.

Finally, the set of kinematically admissible variations is given by (for example, for essential boundary condition):

$$
W_{\Phi}:=\left\{\delta \Phi=(\delta \varphi, \delta \omega) \in \mathbb{R}^{3} \times \mathbb{R}^{3} \mid \delta \boldsymbol{\varphi} \equiv \delta \boldsymbol{\omega} \equiv 0, S \in\{0, L\}\right\}
$$

\subsection{Principle of virtual power}

For a beam structure the kinematics of any point is constrained by the rigid motion of the section. In particular, the displacements $\mathbf{u}$ (respectively $\delta \mathbf{u}$ ) present in Eq. (5) may be written in terms of $\boldsymbol{\varphi}$ and $\mathbf{Q}$ (respectively $\delta \boldsymbol{\varphi}$ and $\delta \boldsymbol{\omega})$ [35]. By definition $\mathbf{u}=\mathbf{O M}-\mathbf{O M}_{\mathbf{0}}$ where $\mathbf{O M}$ is the position in the current configuration, whereas $\mathbf{O M}_{\mathbf{0}}=\varphi_{\mathbf{0}}+\mathbf{G}_{\mathbf{0}} \mathbf{M}_{\mathbf{0}}$ is the position in the reference configuration. In the new configuration $\mathbf{O M}=\mathbf{O G}+\mathbf{G M}$, where $\mathbf{O G}=\boldsymbol{\varphi}$ and $\mathbf{G M}=\mathbf{Q}\left(\mathbf{G}_{\mathbf{0}} \mathbf{M}_{\mathbf{0}}\right)$. Then $\mathbf{u}=\boldsymbol{\varphi}-\boldsymbol{\varphi}_{\mathbf{0}}+(\mathbf{Q}-\mathbf{I}) \mathbf{G}_{\mathbf{0}} \mathbf{M}_{\mathbf{0}}$. Small variations with respect to the kinematics and time differentiation of the beam structure give:

$$
\delta \mathbf{u}=\delta \boldsymbol{\varphi}+\delta \boldsymbol{\omega} \times \mathbf{G M}, \quad \frac{\partial^{2} \mathbf{u}}{\partial t^{2}}=\frac{\partial}{\partial t}\left(\frac{\partial \boldsymbol{\varphi}}{\partial t}+\boldsymbol{\omega} \times \mathbf{G M}\right) .
$$

\subsubsection{External virtual power}

Consider first the external virtual power using the kinematics of rigid body of any section:

$$
\mathcal{P}_{e}(\Phi, \delta \Phi)=\int_{\mathcal{B}} \rho_{0} \mathbf{b} \cdot(\delta \boldsymbol{\varphi}+\delta \boldsymbol{\omega} \times \mathbf{G M}) \mathrm{d} V+\int_{\partial \mathcal{B}} \mathbf{f} \cdot(\delta \boldsymbol{\varphi}+\delta \boldsymbol{\omega} \times \mathbf{G M}) \mathrm{d} A
$$

Using invariance of the scalar triple product under a circular shift, we obtain:

$$
\mathcal{P}_{e}(\Phi, \delta \Phi)=\int_{\mathcal{B}} \delta \boldsymbol{\varphi} \cdot \rho_{0} \mathbf{b}+\delta \boldsymbol{\omega} \cdot\left(\mathbf{G M} \times \rho_{0} \mathbf{b}\right) \mathrm{d} V+\int_{\partial \mathcal{B}} \delta \boldsymbol{\varphi} \cdot \mathbf{f}+\delta \boldsymbol{\omega} \cdot(\mathbf{G M} \times \mathbf{f}) \mathrm{d} A .
$$

On the one hand, we have $\int_{\mathcal{B}} \mathrm{d} V=\int_{\mathcal{C}} \int_{\mathcal{S}} \mathrm{d} A \mathrm{~d} S$ and $\int_{\partial \mathcal{B}} \mathrm{d} A=\int_{\mathcal{C}} \int_{\partial \mathcal{S}} \mathrm{d} L \mathrm{~d} S$, and on the other hand, $\delta \boldsymbol{\varphi}$ and $\delta \omega$ are independent of the local coordinates $\left(\xi_{1}, \xi_{2}\right)$ of the section, they are not affected by the integration over $\mathcal{S}$. We can write:

$$
\mathcal{P}_{e}(\Phi, \delta \Phi)=\int_{\mathcal{C}} \mathbf{q} \cdot \delta \boldsymbol{\varphi}+\mathbf{m} \cdot \delta \boldsymbol{\omega} \mathrm{d} S
$$

in which the external applied force $\mathbf{q}(S, t)$ per unit length is:

$$
\mathbf{q}:=\int_{\mathcal{S}} \rho_{0} \mathbf{b} \mathrm{d} A+\int_{\partial \mathcal{S}} \mathbf{f} \mathrm{d} L
$$

and the external applied couple $\mathbf{m}(S, t)$ per unit reference length is:

$$
\mathbf{m}:=\int_{\mathcal{S}} \mathbf{G M} \times \rho_{0} \mathbf{b} \mathrm{d} A+\int_{\partial \mathcal{S}} \mathbf{G M} \times \mathbf{f} \mathrm{d} L .
$$




\subsubsection{Internal virtual power}

We begin with the internal virtual power in the form given in Eq. (8). Here, the perturbation of the deformation gradient is $\delta \mathbf{F}=\delta\left(\nabla_{X} \mathbf{O M}\right)=\nabla_{X}(\delta \mathbf{O M})$, with $\delta \mathbf{O M}=\delta \mathbf{u}$. In other words:

$$
\delta \mathbf{F}=\left(\delta \boldsymbol{\omega} \times \mathbf{d}_{\alpha}\right) \otimes \mathbf{D}_{\alpha}+\left(\frac{\partial}{\partial S}(\delta \boldsymbol{\varphi}+\delta \boldsymbol{\omega} \times \mathbf{G M})\right) \otimes \mathbf{D}_{\mathbf{3}} .
$$

In order to simplify notations, we introduce $\mathbf{p}_{\mathbf{i}}$ such that $\mathbf{p}_{\mathbf{i}}=\mathbf{P}\left(\mathbf{D}_{\mathbf{i}}\right)$ then $\mathbf{P}=\mathbf{p}_{\mathbf{i}} \otimes \mathbf{D}_{\mathbf{i}}$ (note that $\mathbf{p}_{\mathbf{i}}$ must not been seen as a $i$-component of a vector). With these tools, the density of virtual power becomes:

$$
\begin{aligned}
\mathbf{P}: \delta \mathbf{F} & =\mathbf{p}_{\alpha} \cdot\left(\delta \boldsymbol{\omega} \times \mathbf{d}_{\alpha}\right)+\mathbf{p}_{\mathbf{3}} \cdot\left(\frac{\partial}{\partial S}(\delta \boldsymbol{\varphi}+\delta \boldsymbol{\omega} \times \mathbf{G M})\right) \\
& =\delta \boldsymbol{\omega} \cdot\left(\mathbf{d}_{\alpha} \times \mathbf{p}_{\alpha}\right)+\mathbf{p}_{\mathbf{3}} \cdot\left(\frac{\partial}{\partial S}(\delta \boldsymbol{\varphi}+\delta \boldsymbol{\omega} \times \mathbf{G M})\right) .
\end{aligned}
$$

At this stage, no specific information is explicitly given on $\mathbf{p}_{\alpha}$. However, we have to bear in mind that symmetry of the second Piola-Kirchhoff stress tensor implies $\mathbf{F P}^{T}-\mathbf{P} \mathbf{F}^{T}=\mathbf{0}$. This can be written explicitly as:

$$
\begin{aligned}
\mathbf{d}_{1} \otimes \mathbf{p}_{1} & -\mathbf{p}_{\mathbf{1}} \otimes \mathbf{d}_{\mathbf{1}}+\mathbf{d}_{\mathbf{2}} \otimes \mathbf{p}_{\mathbf{2}}-\mathbf{p}_{\mathbf{2}} \otimes \mathbf{d}_{\mathbf{2}} \\
& +\ldots\left(\frac{\partial \boldsymbol{\varphi}}{\partial S}+\kappa \times \mathbf{G M}\right) \otimes \mathbf{p}_{\mathbf{3}}-\mathbf{p}_{\mathbf{3}} \otimes\left(\frac{\partial \boldsymbol{\varphi}}{\partial S}+\kappa \times \mathbf{G M}\right)=\mathbf{0} .
\end{aligned}
$$

The operation leads to canceling a skew-symmetric tensor. In a trivial way, this is equivalent to canceling its axial vector, and indeed

$$
\mathbf{p}_{\mathbf{1}} \times \mathbf{d}_{\mathbf{1}}+\mathbf{p}_{\mathbf{2}} \times \mathbf{d}_{\mathbf{2}}+\mathbf{p}_{\mathbf{3}} \times\left(\frac{\partial \varphi}{\partial S}+\boldsymbol{\kappa} \times \mathbf{G M}\right)=\mathbf{0},
$$

that gives a relation between $\mathbf{p}_{\alpha}$ and $\mathbf{p}_{\mathbf{3}}$ imposed by the angular momentum conservation law. Introducing this relation in the virtual internal power density Eq. (26):

$$
\mathbf{P}: \delta \mathbf{F}=\delta \boldsymbol{\omega} \cdot\left(\mathbf{p}_{\mathbf{3}} \times\left(\frac{\partial \boldsymbol{\varphi}}{\partial S}+\boldsymbol{\kappa} \times \mathbf{G M}\right)\right)+\mathbf{p}_{\mathbf{3}} \cdot\left(\frac{\partial}{\partial S}(\delta \boldsymbol{\varphi}+\delta \boldsymbol{\omega} \times \mathbf{G M})\right) .
$$

Expanding each term, we obtain:

$$
\mathbf{P}: \delta \mathbf{F}=\mathbf{p}_{\mathbf{3}} \cdot\left(\frac{\partial \delta \boldsymbol{\varphi}}{\partial S}-\delta \boldsymbol{\omega} \times \frac{\partial \boldsymbol{\varphi}}{\partial S}\right)+\left(\mathbf{G M} \times \mathbf{p}_{\mathbf{3}}\right) \cdot \frac{\partial \delta \boldsymbol{\omega}}{\partial S} .
$$

For the internal power written in terms of $\mathbf{P}: \delta \mathbf{F}$, it is necessary to remind that variation of strain is frameindifferent, e.g., [38]. Corotational variation must be prescribed. For any vector $\mathbf{a}=a_{i} \mathbf{d}_{\mathbf{i}}$, this variation is:

$$
\delta^{R} \mathbf{a}:=\delta \mathbf{a}-\delta \boldsymbol{\omega} \times \mathbf{a}=\left(\delta a_{i}\right) \mathbf{d}_{\mathbf{i}} .
$$

These corotational variations are well defined in Eq. (27) and closely related to Maurer-Cartan form; indeed according to Eqs. (11) and (12):

$$
\delta^{R} \boldsymbol{\varepsilon}=\frac{\partial \delta \boldsymbol{\varphi}}{\partial S}-\delta \boldsymbol{\omega} \times \frac{\partial \boldsymbol{\varphi}}{\partial S}, \quad \delta^{R} \boldsymbol{\kappa}=\delta \boldsymbol{\kappa}-\delta \boldsymbol{\omega} \times \boldsymbol{\kappa}=\frac{\partial \delta \boldsymbol{\omega}}{\partial S} .
$$

These corotational variations are uniform along the beam section and are not affected by integration over the beam section. Hence, according to Eq. (17) (with $\mathbf{P}\left(\mathbf{D}_{\mathbf{3}}\right)=\mathbf{p}_{\mathbf{3}}$ ), this integration makes appear the force and torque per unit length. Then, virtual internal power becomes [24]:

$$
\mathcal{P}_{i}(\Phi, \delta \Phi)=\int_{\mathcal{C}} \mathrm{N} \cdot \delta^{R} \boldsymbol{\varepsilon}+\mathrm{M} \cdot \delta^{R} \boldsymbol{\kappa} \mathrm{d} S .
$$

In other words, for beam structures, the dual quantities associated with forces and torques per unit length are corotational deformation and curvature, respectively. 


\subsubsection{Remark}

Using Eq. (9) gives some new information. For example, in the case of a hyperelastic material $\mathbf{S}$ is related to the Helmholtz free energy $\psi(\mathbf{E})$ :

$$
\mathbf{S}=\frac{\partial \psi}{\partial \mathbf{E}}, \quad \text { or } \quad S_{I J}=\frac{\partial \psi}{\partial E_{I J}} .
$$

In such a case, $\mathrm{C}$ has a major symmetry $\mathrm{C}_{I J K L}=\mathrm{C}_{K L I J}$. We may observe that

$$
\delta E_{I J}=\frac{\partial E_{I J}}{\partial \varepsilon_{l}} \delta \varepsilon_{l}+\frac{\partial E_{I J}}{\partial \kappa_{l}} \delta \kappa_{l}
$$

where $\kappa_{i}$ and $\varepsilon_{i}$ are components of the strain vectors in the current configuration: $\boldsymbol{\kappa}=\kappa_{i} \mathbf{d}_{\mathbf{i}}$ and $\boldsymbol{\varepsilon}=\varepsilon_{i} \mathbf{d}_{\mathbf{i}}$. Hence, we can easily compute the virtual power:

$$
\mathcal{P}_{i}(\Phi, \delta \Phi)=\int_{\mathcal{B}} \mathbf{S}: \delta \mathbf{E} \mathrm{d} V=\int_{\mathcal{C}} \int_{\mathcal{S}} \frac{\partial \psi}{\partial \varepsilon_{i}} \delta \varepsilon_{i}+\frac{\partial \psi}{\partial \kappa_{i}} \delta \kappa_{i} \mathrm{~d} A \mathrm{~d} S .
$$

Direct identification with Eq. (29) gives the components on the $\left\{\mathbf{d}_{\mathbf{i}}\right\}$-basis:

$$
N_{i}=\int_{\mathcal{S}} \frac{\partial \psi}{\partial \varepsilon_{i}} \mathrm{~d} A, \quad M_{i}=\int_{\mathcal{S}} \frac{\partial \psi}{\partial \kappa_{i}} \mathrm{~d} A .
$$

Because $\varepsilon_{i}$ and $\kappa_{i}$ are uniform on the section $\mathcal{S}$, the integration and derivation may be switched to give:

$$
\mathbf{N}=\frac{\partial \Psi}{\partial \varepsilon_{i}} \mathbf{d}_{\mathbf{i}}, \quad \mathbf{M}=\frac{\partial \Psi}{\partial \kappa_{i}} \mathbf{d}_{\mathbf{i}},
$$

where $\Psi$ is the free energy density per unit length of the beam structure:

$$
\Psi:=\int_{\mathcal{S}} \psi \mathrm{d} A
$$

This formulation is valid for any hyperelastic models.

\subsubsection{Virtual power of inertia}

According to the kinematical variables, the virtual power of inertia Eq. (6) takes the following form:

$$
\mathcal{P}_{j}(\Phi, \delta \Phi)=\int_{\mathcal{C}} \int_{\mathcal{S}} \rho_{0} \frac{\partial}{\partial t}\left(\frac{\partial \boldsymbol{\varphi}}{\partial t}+\boldsymbol{\omega} \times \mathbf{G M}\right) \cdot(\delta \boldsymbol{\varphi}+\delta \boldsymbol{\omega} \times \mathbf{G M}) \mathrm{d} A \mathrm{~d} S .
$$

Of course, $\int_{\mathcal{S}} \mathbf{G M} \mathrm{d} A=\mathbf{0}$. Moreover, in order to simplify computation, we consider uniform cross section, and then $\int_{\mathcal{S}} \rho_{0} \mathrm{~d} A=\rho_{0} A$. Remembering the relation and the double vector product $\mathbf{a} \times(\mathbf{b} \times \mathbf{c})=(\mathbf{a} \cdot \mathbf{c}) \mathbf{b}-(\mathbf{a} \cdot \mathbf{b}) \mathbf{c}$, we obtain the following relation:

$$
\mathcal{P}_{j}(\Phi, \delta \Phi)=\int_{\mathcal{C}} \rho_{0} A \frac{\partial^{2} \boldsymbol{\varphi}}{\partial t^{2}} \cdot \delta \boldsymbol{\varphi}+\frac{\partial}{\partial t}\left[\int_{\mathcal{S}} \rho_{0}(\mathbf{G M} \times(\omega \times \mathbf{G M})) \mathrm{d} A\right] \cdot \delta \boldsymbol{\omega} \mathrm{d} S .
$$

Introducing $\mathbb{I}$, the tensor of quadratic moment of the section, we have for any vector a

$$
\int_{\mathcal{S}} \mathbf{G M} \times(\mathbf{a} \times \mathbf{G M}) \mathrm{d} A=\mathbb{I} \mathbf{a} .
$$

For uniform mass density in the section, the virtual power of inertia becomes:

$$
\mathcal{P}_{j}(\Phi, \delta \Phi)=\int_{\mathcal{C}} \rho_{0} A \frac{\partial^{2} \boldsymbol{\varphi}}{\partial t^{2}} \cdot \delta \varphi+\rho_{0} \frac{\partial}{\partial t}(\mathbb{I} \boldsymbol{\omega}) \cdot \delta \boldsymbol{\omega} \mathrm{d} S .
$$

If $\left\{\mathbf{d}_{\mathbf{i}}\right\}$ denotes the principal basis of the section, the tensor of quadratic moment is diagonal $\mathbb{I}=\sum_{i=1}^{3} I_{i} \mathbf{d}_{\mathbf{i}} \otimes \mathbf{d}_{\mathbf{i}}$, with

$$
I_{1}=\int_{\mathcal{S}} \xi_{2}^{2} \mathrm{~d} A, \quad I_{2}=\int_{\mathcal{S}} \xi_{1}^{2} \mathrm{~d} A, \quad I_{3}=\int_{\mathcal{S}}\left(\xi_{1}^{2}+\xi_{2}^{2}\right) \mathrm{d} A
$$


4.3 Principle of virtual power in Lagrangian form for large transformation

The principle of virtual power gives us the variational equation of a beam:

$$
\mathcal{P}_{j}(\Phi, \delta \Phi)+\mathcal{P}_{i}(\Phi, \delta \Phi)=\mathcal{P}_{e}(\Phi, \delta \Phi) .
$$

Considering a bounded beam of length $\ell$, we have after integrating by parts:

$$
\mathcal{P}_{i}(\Phi, \delta \Phi)=[\mathrm{N} \cdot \delta \varphi+\mathrm{M} \cdot \delta \omega]_{0}^{\ell}-\int_{\mathcal{C}} \frac{\partial \mathrm{N}}{\partial S} \cdot \delta \varphi+\left(\frac{\partial \varphi}{\partial S} \times \mathrm{N}+\frac{\partial \mathrm{M}}{\partial S}\right) \cdot \delta \omega \mathrm{d} S .
$$

Using the previous results, we obtain $\forall(\delta \varphi, \delta \omega) \in W_{\Phi}$ :

$$
\begin{aligned}
{[\mathrm{N} \cdot \delta \boldsymbol{\varphi}+\mathrm{M} \cdot \delta \boldsymbol{\omega}]_{0}^{\ell}=} & \int_{\mathcal{C}}\left(\frac{\partial \mathrm{N}}{\partial S}+\mathbf{q}-\rho_{0} A \frac{\partial^{2} \boldsymbol{\varphi}}{\partial t^{2}}\right) \cdot \delta \boldsymbol{\varphi} \mathrm{d} S \\
& +\int_{\mathcal{C}}\left(\frac{\partial \mathrm{M}}{\partial S}+\frac{\partial \boldsymbol{\varphi}}{\partial S} \times \mathrm{N}+\mathbf{m}-\rho_{0} \frac{\partial}{\partial t}(\mathbb{I} \boldsymbol{\omega})\right) \cdot \delta \boldsymbol{\omega} \mathrm{d} S .
\end{aligned}
$$

This equation may help to obtain the strong formulation of the dynamic equation of the beam structure under finite transformation. It must be emphasized that this formulation is equivalent to the case of infinitesimal transformation. This is mainly due to the choice of adapted kinematical variable.

\section{Superimposed wave}

Suppose that the beam undergoes a prescribed finite motion $\Phi$. The main objective of this section is to define the equation satisfied by small vibrations $\Delta \Phi$ superimposed on this pre-stressed configuration in the absence of additional external excitation.

Of course, in the presence of this superimposed vibration, the principe of virtual power Eq. (35) must still be satisfied for any $(\delta \varphi, \delta \omega) \in W_{\Phi}$ :

$$
\mathcal{P}_{j}(\Phi+\Delta \Phi, \delta \Phi)+\mathcal{P}_{i}(\Phi+\Delta \Phi, \delta \Phi)=\mathcal{P}_{e}(\Phi+\Delta \Phi, \delta \Phi) .
$$

For an infinitesimal superimposed wave, the perturbation of the virtual power (for example for the virtual power of inertia),

$$
\Delta \mathcal{P}_{j}(\Phi, \delta \Phi)=\mathcal{P}_{j}(\Phi+\Delta \Phi, \delta \Phi)-\mathcal{P}_{j}(\Phi, \delta \Phi),
$$

can be approached by linearization:

$$
\Delta \mathcal{P}_{j}(\Phi, \delta \Phi)=D \mathcal{P}_{j}(\Phi, \delta \Phi)[\Delta \Phi]
$$

The main difficulty is then to give a rigorous formulation of these second order variations. In fact $\Delta \mathcal{P}_{j}(\Phi, \delta \Phi)$ is an increment associated with a Gâteau-Fréchet derivative:

$$
D \mathcal{P}_{j}(\Phi, \delta \Phi)[\Delta \Phi]:=\lim _{h \rightarrow 0}\left(\frac{1}{h}\left(\mathcal{P}_{j}(\Phi+h \Delta \Phi, \delta \Phi)-\mathcal{P}_{j}(\Phi, \delta \Phi)\right)\right)[\Delta \Phi]
$$

The same is done for the internal and external virtual power. Because Eq. (35) is still satisfied, we have to prescribe the following relation:

$$
\Delta \mathcal{P}_{j}(\Phi, \delta \Phi)+\Delta \mathcal{P}_{i}(\Phi, \delta \Phi)=\Delta \mathcal{P}_{e}(\Phi, \delta \Phi)
$$

Each of these terms are explicitly computed in the following. 


\subsection{Inertia}

Using the definition of the second variation, we have

$$
\begin{aligned}
\Delta \mathcal{P}_{j}(\Phi, \delta \Phi) & =\lim _{h \rightarrow 0}\left(\frac{1}{h}\left(\int_{\mathcal{B}} \rho_{0} \frac{\partial^{2} \mathbf{u}+h \Delta \mathbf{u}}{\partial t^{2}} \cdot \delta \mathbf{u} \mathrm{d} V-\int_{\mathcal{B}} \rho_{0} \frac{\partial^{2} \mathbf{u}}{\partial t^{2}} \cdot \delta \mathbf{u} \mathrm{d} V\right)\right) \\
& =\int_{\mathcal{B}} \rho_{0} \frac{\partial^{2} \Delta \mathbf{u}}{\partial t^{2}} \cdot \delta \mathbf{u d} V .
\end{aligned}
$$

The previous calculation done for the first variation gives directly:

$$
\Delta \mathcal{P}_{j}(\Phi, \delta \Phi)=\int_{\mathcal{C}} \delta \Phi^{T} \mathbb{M} \Delta \Phi \mathrm{d} S
$$

where $\mathbb{M}(\Phi)$ is the tangent inertial operator of the beam:

$$
\mathbb{M}:=\left(\begin{array}{cc}
\rho_{0} A \frac{\partial^{2}}{\partial t^{2}} & 0 \\
0 & \rho_{0} \frac{\partial}{\partial t} \mathbb{I}
\end{array}\right)
$$

\subsection{External virtual power}

For the external virtual power, the linearization gives:

$$
\begin{aligned}
\Delta \mathcal{P}_{e}(\Phi, \delta \Phi)= & \lim _{h \rightarrow 0}\left(\frac{1}{h}\left(\mathcal{P}_{e}(\Phi+h \Delta \Phi, \delta \Phi)-\mathcal{P}_{e}(\Phi, \delta \Phi)\right)\right), \\
= & \int_{\mathcal{B}} \rho_{0}(\Delta \mathbf{b} \cdot(\delta \boldsymbol{\varphi}+\delta \boldsymbol{\omega} \times \mathbf{G M})+\mathbf{b} \cdot(\delta \boldsymbol{\omega} \times \Delta \mathbf{G M})) \mathrm{d} V \\
& +\ldots \int_{\partial \mathcal{B}} \Delta \mathbf{f} \cdot(\delta \boldsymbol{\varphi}+\delta \boldsymbol{\omega} \times \mathbf{G M})+\mathbf{f} \cdot(\delta \boldsymbol{\omega} \times \Delta \mathbf{G M}) \mathrm{d} A .
\end{aligned}
$$

On the one hand, $\Delta \mathbf{G M}=\Delta \boldsymbol{\omega} \times \mathbf{G M}$, and on the other hand, it must be reminded that the perturbation of the force density reads

$$
\Delta \mathbf{b}=\Delta^{R} \mathbf{b}+\Delta \omega \times \mathbf{b}
$$

with $\Delta^{R} \mathbf{b}=\left(\Delta b_{i}\right) \mathbf{d}_{\mathbf{i}}$.

For example, for a constant dead load $\Delta \mathbf{b}=0$ (then $\Delta^{R} \mathbf{b}=-\Delta \omega \times \mathbf{b}$ ), and for a constant follower load $\Delta^{R} \mathbf{b}=0$ (then $\Delta \mathbf{b}=\Delta \boldsymbol{\omega} \times \mathbf{b}$ ). The same holds true for $\mathbf{f}$. A priori it is possible to write the internal power in two different but equivalent ways. Focusing first on the first term in Eq. (37):

$$
\begin{aligned}
\Delta \mathbf{b} \cdot(\delta \boldsymbol{\varphi}+ & \delta \boldsymbol{\omega} \times \mathbf{G M})+\mathbf{b} \cdot(\delta \boldsymbol{\omega} \times \Delta \mathbf{G M}) \\
\quad= & \left\{\begin{array}{l}
\Delta \mathbf{b} \cdot \delta \boldsymbol{\varphi}+(\mathbf{G M} \times \Delta \mathbf{b}+(\Delta \boldsymbol{\omega} \times \mathbf{G M}) \times \mathbf{b}) \cdot \delta \boldsymbol{\omega}, \\
\left(\Delta^{R} \mathbf{b}+\Delta \boldsymbol{\omega} \times \mathbf{b}\right) \cdot \delta \boldsymbol{\varphi}+\left(\mathbf{G M} \times \Delta^{R} \mathbf{b}+\Delta \boldsymbol{\omega} \times(\mathbf{b} \times \mathbf{G M})\right) \cdot \delta \boldsymbol{\omega}
\end{array}\right.
\end{aligned}
$$

where the Jacobi identity has been used. For the second term, we have:

$$
\begin{aligned}
\Delta \mathbf{f} \cdot(\delta \boldsymbol{\varphi}+ & \delta \boldsymbol{\omega} \times \mathbf{G M})+\mathbf{f} \cdot(\delta \boldsymbol{\omega} \times \Delta \mathbf{G M}) \\
& =\left\{\begin{array}{l}
\Delta \mathbf{f} \cdot \delta \boldsymbol{\varphi}+(\mathbf{G M} \times \Delta \mathbf{f}+(\Delta \boldsymbol{\omega} \times \mathbf{G M}) \times \mathbf{f}) \cdot \delta \boldsymbol{\omega}, \\
\left(\Delta^{R} \mathbf{f}+\Delta \boldsymbol{\omega} \times \mathbf{f}\right) \cdot \delta \boldsymbol{\varphi}+\left(\mathbf{G M} \times \Delta^{R} \mathbf{f}+\Delta \boldsymbol{\omega} \times(\mathbf{f} \times \mathbf{G M})\right) \cdot \delta \boldsymbol{\omega} .
\end{array}\right.
\end{aligned}
$$

If we use for example the first formulation, we have after integration:

$$
\Delta \mathcal{P}_{e}(\Phi, \delta \Phi)=\int_{\mathcal{S}} \Delta \mathbf{q} \cdot \delta \boldsymbol{\varphi}+\Delta \mathbf{m} \cdot \delta \boldsymbol{\omega} \mathrm{d} S
$$


with

$$
\Delta \mathbf{q}:=\int_{\mathcal{S}} \rho_{0} \Delta \mathbf{b} \mathrm{d} A+\int_{\partial \mathcal{S}} \Delta \mathbf{f} \mathrm{d} L
$$

and

$$
\Delta \mathbf{m}:=\int_{\mathcal{S}}(\mathbf{G M} \times \Delta \mathbf{b}+(\Delta \omega \times \mathbf{G M}) \times \mathbf{b}) \mathrm{d} A+\int_{\partial \mathcal{S}}(\mathbf{G M} \times \Delta \mathbf{f}+(\Delta \omega \times \mathbf{G M}) \times \mathbf{f}) \mathrm{d} L .
$$

In order to illustrate the extremely different effect of dead or follower load, we analyze here two simplified cases:

- Constant dead load $\Delta \mathbf{b}=0$. In such a case, the perturbation of the external power related to this force density becomes

$$
\Delta \mathcal{P}_{e}(\Phi, \delta \Phi)=\int_{\mathcal{B}} \rho_{0}((\Delta \omega \times \mathbf{G M}) \times \mathbf{b}) \cdot \delta \omega \mathrm{d} V
$$

However, if $\mathbf{b}$ is uniform on the cross section, the integration around the section induces

$$
\Delta \mathcal{P}_{e}(\Phi, \delta \Phi)=0
$$

because $\int_{\mathcal{S}} \rho_{0} \mathbf{G M} \mathrm{d} A=0$.

- Constant follower load $\Delta^{R} \mathbf{b}=0$. In such a case the perturbation of the external power related to this force density becomes

$$
\Delta \mathcal{P}_{e}(\Phi, \delta \Phi)=\int_{\mathcal{B}} \rho_{0}((\Delta \omega \times \mathbf{b}) \cdot \delta \boldsymbol{\varphi}+(\Delta \omega \times(\mathbf{b} \times \mathbf{G M})) \cdot \delta \boldsymbol{\omega}) \mathrm{d} V .
$$

Again if the pre-stress is uniform, we obtain after integration over the cross section:

$$
\Delta \mathcal{P}_{e}(\Phi, \delta \Phi)=\int_{\mathcal{C}} \rho_{0} A(\Delta \omega \times \mathbf{b}) \cdot \delta \boldsymbol{\varphi} \mathrm{d} S,
$$

that does not vanish if the angular vibration is not orthogonal to the load.

In this example, we do not introduces $\mathbf{f}$ for sake of the simplicity, but the same analysis can be performed without difficulties. These two examples show how the type of load may introduce some additional external power according to the geometrical and material properties of the load.

\subsection{Internal virtual power}

For the internal virtual power, the linearization leads to

$$
\Delta \mathcal{P}_{i}(\Phi, \delta \Phi)=\lim _{h \rightarrow 0}\left(\frac{1}{h}\left(\mathcal{P}_{i}(\Phi+h \Delta \Phi, \delta \Phi)-\mathcal{P}_{i}(\Phi, \delta \Phi)\right)\right) .
$$

In other words, we have to compute the tangent operator of the virtual power density, i.e., the linear variation of (keeping the variation $\delta$ constant):

$$
\Delta \mathcal{P}_{i}(\Phi, \delta \Phi)=\Delta \int_{\mathcal{B}} \mathbf{P}: \delta \mathbf{F} \mathrm{d} V
$$

Because the integration is performed on the beam in the reference configuration, the linearization acts only on the term in the integral:

$$
\Delta \mathcal{P}_{i}(\Phi, \delta \Phi)=\int_{\mathcal{B}}(\Delta \mathbf{P}): \delta \mathbf{F} \mathrm{d} V
$$


Bearing in mind $\mathbf{P}=\mathbf{F S}$, we have $\Delta \mathbf{P}=(\Delta \mathbf{F}) \mathbf{S}+\mathbf{F}(\Delta \mathbf{S})$ and obtain:

$$
\Delta \mathcal{P}_{i}(\Phi, \delta \Phi)=\int_{\mathcal{B}}(\Delta \mathbf{F}) \mathbf{S}: \delta \mathbf{F}+(\mathbf{F}(\Delta \mathbf{S})): \delta \mathbf{F} \mathrm{d} V
$$

The first term, $\mathbf{S}:\left(\Delta \mathbf{F}^{T} \delta \mathbf{F}\right)$ (or explicitly $\left.\Delta F_{i I} S_{I J} \delta F_{i J}\right)$, is entirely symmetric. For the second term, this is less clear, and some manipulations are needed and presented below.

As it has been prescribed earlier, the expression of $S_{I J}$ may depend one the material response of the medium, but the relation between $\Delta S_{J I}=\Delta S_{I J}$ and $\Delta E_{K L}$ may be written according to the elastic tensor computed at the pre-deformed configuration. Indeed, Eq. (2) is seen with a perturbation point of view (but without any approximation) as:

$$
\Delta S_{I J}=\mathrm{C}_{I J K L} \Delta E_{K L}
$$

where $\mathrm{C}$ is still evaluated around the $\boldsymbol{\Phi}$-state. Because

$$
\Delta E_{K L}=\frac{1}{2}\left(\Delta F_{j K} F_{j L}+F_{j K} \Delta F_{j L}\right)
$$

we obtain

$$
F_{i I} \Delta S_{I J} \delta F_{i J}=\frac{1}{2} F_{i I} \delta F_{i J} \mathrm{C}_{I J K L}\left(\Delta F_{j K} F_{j L}+F_{j K} \Delta F_{j L}\right) .
$$

Moreover, right minor symmetry of the elasticity tensor, $\mathrm{C}_{I J K L}=\mathrm{C}_{I J L K}$, may be used. Indeed, $\mathrm{C}_{I J K L} F_{j K} \Delta F_{j L}=\mathrm{C}_{I J L K} F_{j K} \Delta F_{j L}$, and by interchanging the indices names, $K \leftrightarrow L$, we finally obtain $\mathrm{C}_{I J K L} F_{j L} \Delta F_{j K}$. Insertion of this relation into the previous equation leads to the summation of two identical terms, and then:

$$
F_{i I} \Delta S_{I J} \delta F_{i J}=F_{i I} \delta F_{i J} \mathrm{C}_{I J K L} \Delta F_{j K} F_{j L}
$$

that may be written as

$$
\left(\mathbf{F}^{T} \delta \mathbf{F}\right): \mathbf{C}:\left(\mathbf{F}^{T} \Delta \mathbf{F}\right)=\delta \mathbf{E}: \mathbf{C}(\Delta \mathbf{E}) .
$$

The tangent operator is then

$$
\Delta \mathcal{P}_{i}(\Phi, \delta \Phi)=\int_{\mathcal{B}} \mathbf{S}:\left(\Delta \mathbf{F}^{T} \delta \mathbf{F}\right)+\delta \mathbf{E}: \mathbf{C}(\Delta \mathbf{E}) \mathrm{d} V
$$

This is one of the main equations of the present paper. The general character of this equation must be pointed out as it does not use any hypotheses on the mechanical response of the material.

First we observe that the equation is entirely symmetric (with respect to $\delta$ and $\Delta$ transformations) that ensures a self-adjoint operator.

The first term may be interpreted as a geometric contribution, whereas the second one is the material contribution. Both are quadratic in terms of $\mathbf{F}$. For finite transformation material and geometrical contribution are of the same order of magnitude. In the absence of finite pre-transformation, $\mathbf{F}=\mathbf{I}$ (identity), and according to Eq. (39) the strain is the standard small strain tensor. Moreover, $\mathbf{S}=\mathbf{0}$ : the wave motion is only governed by the material contribution in absence of pre-stress. For a small pre-stress, we can consider that the geometric contribution is negligible with regard to the full material one.

Integration over the beam section in Eq. (40) may not be performed easily, as section coordinates $\left(\xi_{1}, \xi_{2}\right)$ are present both in $\mathbf{S}$ and $\mathbf{F}, \delta \mathbf{F}, \Delta \mathbf{F}$. From a general point of view, the dependence on $\left(\xi_{1}, \xi_{2}\right)$ is of degree four. An other strong difficulty arises if the pre-stress configuration is time dependent (for time-dependent external loading for example). In such a case the characteristic frequency of the large transformation must be smaller (slow motion) than for the frequencies of vibrations. 


\subsection{General methodology}

In order to solve the general problem, we must notice that the superimposed state $\Delta \Phi$ is related by the motion $\Delta \mathbf{O M}=\Delta \mathbf{u}$ of any point of the beam section. Because the section is rigid this displacement is described by the rotation $\Delta \omega$ of the beam section and the displacement $\Delta \varphi$ of the center of mass: $\Delta \mathbf{u}=\Delta \varphi+\Delta \omega \times \mathbf{G M}$. These two vectors are the solutions of the variational problem Eq. (36)

$$
\int_{\mathcal{B}} \mathbf{S}:\left(\Delta \mathbf{F}^{T} \delta \mathbf{F}\right)+\delta \mathbf{E}: \mathbf{C}(\Delta \mathbf{E}) \mathrm{d} V+\int_{\mathcal{S}} \rho_{0} A \frac{\partial^{2} \Delta \boldsymbol{\varphi}}{\partial t^{2}} \cdot \delta \boldsymbol{\varphi}+\rho_{0} \frac{\partial}{\partial t}(\mathbb{I} \Delta \boldsymbol{\omega}) \cdot \delta \boldsymbol{\omega} \mathrm{d} S=\int_{\mathcal{S}} \Delta \mathbf{q} \cdot \delta \boldsymbol{\varphi}+\Delta \mathbf{m} \cdot \delta \boldsymbol{\omega} \mathrm{d} S .
$$

This fundamental equation may be explicitly written in terms of unknowns $\Delta \varphi$ and $\Delta \omega$. Indeed, after some integration by parts, we obtain a problem of the form: find $\Delta \varphi, \Delta \omega, \forall(\delta \varphi, \delta \omega) \in W_{\Phi}$ such that

$$
\int_{\mathcal{S}}\left(\mathcal{F}(\Delta \varphi, \Delta \omega ; \Phi)-\rho_{0} A \frac{\partial^{2} \Delta \varphi}{\partial t^{2}}\right) \cdot \delta \varphi+\left(\mathcal{M}(\Delta \varphi, \Delta \omega ; \Phi)-\rho_{0} \frac{\partial}{\partial t}(\mathbb{I} \Delta \omega)\right) \cdot \delta \omega \mathrm{d} S=C .
$$

$C$ is the constant obtained after integration by parts and depends on the prescribed load and boundary conditions. $\mathcal{F}$ and $\mathcal{M}$ depend on the external incremental loads $\Delta \mathbf{q}$ and $\Delta \mathbf{m}$, too.

Solving the problem $C=0$ leads to the definition of the essential and natural boundary conditions for the superimposed wave according to the finite transformation. In such a case the resolution of the previous weak problem can be replaced by the resolution of the system of equations parametrized by the large transformation $\Phi$,

$$
\begin{gathered}
\mathcal{F}(\Delta \varphi, \Delta \omega ; \Phi)=\rho_{0} A \frac{\partial^{2} \Delta \varphi}{\partial t^{2}}, \\
\mathcal{M}(\Delta \varphi, \Delta \omega ; \Phi)=\rho_{0} \frac{\partial}{\partial t}(\mathbb{I} \Delta \omega),
\end{gathered}
$$

which are nothing else than the (translational and rotational) equations of motion of the superimposed wave.

Of course, general resolution cannot be performed analytically, and for this reason, we focus in the following on a simple material and then on some specific finite transformation.

\section{Application for a hyperelastic material}

We give a simple application of the previous general methodology. First the material is supposed to be a Kirchhoff-Saint Venant hyperelastic material, second the geometric and material contribution of the internal power is developed in detail.

\subsection{Kirchhoff-Saint Venant hyperelastic material}

In order to give an explicit formulation of the general result previously presented, we consider a Kirchhoff-Saint Venant model of isotropic material for which the elasticity tensor is uniform and defined as:

$$
\mathrm{C}_{I J K L}=\frac{\partial^{2} \psi}{\partial E_{I J} \partial E_{K L}}=\lambda \delta_{I J} \delta_{K L}+\mu\left(\delta_{I K} \delta_{J L}+\delta_{I L} \delta_{J K}\right)
$$

where $\lambda$ and $\mu$ are Lamé's coefficients. It is a special class of (hyper)-elastic material (Eq. (30)) for which the material response is linear:

$$
\mathbf{S}=\mathrm{C} \mathbf{E} \quad \text { or } \quad S_{I J}=\mathrm{C}_{I J K L} E_{K L} .
$$

The continuum Helmholtz free energy is quadratic with regards to the Green-Lagrange strain tensor $\mathbf{E}$ (Eq. (15)):

$$
\psi(\mathbf{E}):=\frac{1}{2} \mathbf{E}: \mathbf{C}(\mathbf{E})=\frac{\lambda}{2}(\operatorname{Tr}(\mathbf{E}))^{2}+\mu \operatorname{Tr}\left(\mathbf{E}^{2}\right) .
$$

For this material, the second Piola-Kirchhoff stress becomes:

$$
\mathbf{S}:=\mathbf{C}(\mathbf{E})=\frac{\partial \psi}{\partial \mathbf{E}}=\lambda \operatorname{Tr}(\mathbf{E}) \mathbf{I}+2 \mu \mathbf{E} .
$$




\subsection{Material and geometric contribution}

The evaluation of the material and the geometric contribution may be performed by using symbolic computation. The key point is that the finite transformation is entirely defined by one vector: $\frac{\partial \mathbf{O M}}{\partial S}$ that simplifies the symbolic computation. Hence, the evaluation of the material and the geometric contribution may be written as:

$$
\begin{aligned}
\mathbf{S}:\left(\Delta \mathbf{F}^{T} \delta \mathbf{F}\right) & =\frac{1}{2} \Delta F_{i I} \mathrm{C}_{I J K L}\left(F_{j K} F_{j L}-\delta_{K L}\right) \delta F_{i J}, \\
\delta \mathbf{E}: \mathbf{C}(\Delta \mathbf{E}) & =F_{i I} \delta F_{i J} \mathrm{C}_{I J K L} \Delta F_{j K} F_{j L}
\end{aligned}
$$

where the components of the elasticity tensor are given in Eq. (42). The next step is to give the matrix notation of $\mathbf{F}$ and its perturbation $\delta \mathbf{F}$ from Eq. (25) (for $\Delta \mathbf{F}$ the same is done-not reported) in the basis $\left(\mathbf{d}_{\mathbf{i}} \otimes \mathbf{D}_{\mathbf{J}}\right)$ :

$$
\mathbf{F}=\left(\begin{array}{lll}
1 & 0 & \mathbf{d}_{\mathbf{1}} \cdot \frac{\partial \mathbf{O M}}{\partial S} \\
0 & 1 & \mathbf{d}_{\mathbf{2}} \cdot \frac{\partial \mathbf{O M}}{\partial S} \\
0 & 0 & \mathbf{d}_{\mathbf{3}} \cdot \frac{\partial \mathbf{O M}}{\partial S}
\end{array}\right), \quad \delta \mathbf{F}=\left(\begin{array}{ccc}
0 & \mathbf{d}_{\mathbf{1}} \cdot\left(\delta \boldsymbol{\omega} \times \mathbf{d}_{\mathbf{2}}\right) \mathbf{d}_{\mathbf{1}} \cdot \frac{\partial \delta \mathbf{u}}{\partial S} \\
\mathbf{d}_{\mathbf{2}} \cdot\left(\boldsymbol{\delta} \boldsymbol{\omega} \times \mathbf{d}_{\mathbf{1}}\right) & 0 & \mathbf{d}_{\mathbf{2}} \cdot \frac{\partial \delta \mathbf{u}}{\partial S} \\
\mathbf{d}_{\mathbf{3}} \cdot\left(\boldsymbol{\delta} \boldsymbol{\omega} \times \mathbf{d}_{\mathbf{1}}\right) & \mathbf{d}_{\mathbf{3}} \cdot\left(\delta \boldsymbol{\delta} \times \mathbf{d}_{\mathbf{2}}\right) \mathbf{d}_{\mathbf{3}} \cdot \frac{\partial \delta \mathbf{u}}{\partial S}
\end{array}\right) .
$$

We respectively obtain for the geometric contribution:

$$
\begin{aligned}
\mathbf{S}:\left(\Delta \mathbf{F}^{T} \delta \mathbf{F}\right)= & \left(\frac{\lambda}{2}+\mu\right)\left(\left\|\frac{\partial \mathbf{O M}}{\partial S}\right\|^{2}-1\right) \frac{\partial \delta \mathbf{u}}{\partial S} \cdot \frac{\partial \Delta \mathbf{u}}{\partial S} \\
& +\frac{\lambda}{2}\left(\left\|\frac{\partial \mathbf{O M}}{\partial S}\right\|^{2}-1\right)\left(\delta \boldsymbol{\omega} \times \mathbf{d}_{\alpha}\right) \cdot\left(\Delta \boldsymbol{\omega} \times \mathbf{d}_{\alpha}\right) \\
& +\mu\left(\mathbf{d}_{\alpha} \cdot \frac{\partial \mathbf{O M}}{\partial S}\right)\left(\mathbf{d}_{\alpha} \cdot\left(\frac{\partial \delta \mathbf{u}}{\partial S} \times \Delta \boldsymbol{\omega}+\frac{\partial \Delta \mathbf{u}}{\partial S} \times \delta \boldsymbol{\omega}\right)\right),
\end{aligned}
$$

and for the material contribution:

$$
\begin{aligned}
\delta \mathbf{E}: \mathbf{C}(\Delta \mathbf{E})= & \mu\left(\mathbf{d}_{\alpha} \cdot\left(\frac{\partial \Delta \mathbf{u}}{\partial S}-\Delta \boldsymbol{\omega} \times \frac{\partial \mathbf{O M}}{\partial S}\right)\right)\left(\mathbf{d}_{\alpha} \cdot\left(\frac{\partial \delta \mathbf{u}}{\partial S}-\delta \boldsymbol{\omega} \times \frac{\partial \mathbf{O M}}{\partial S}\right)\right) \\
& +(\lambda+2 \mu)\left(\frac{\partial \mathbf{O M}}{\partial S} \cdot \frac{\partial \Delta \mathbf{u}}{\partial S}\right)\left(\frac{\partial \mathbf{O M}}{\partial S} \cdot \frac{\partial \delta \mathbf{u}}{\partial S}\right) .
\end{aligned}
$$

The integration by parts is not trivial; for example, we have to keep in mind that:

$$
\frac{\partial \delta \mathbf{u}}{\partial S}=\frac{\partial \delta \boldsymbol{\varphi}}{\partial S}+\frac{\partial \delta \boldsymbol{\omega}}{\partial S} \times \mathbf{G M}+\delta \boldsymbol{\omega} \times(\boldsymbol{\kappa} \times \mathbf{G M})
$$

which is due to the $S$ dependence of the local frame $\mathbf{d}_{\mathbf{i}}$ (as the curvature induced by the initial transformation $\Phi)$.

\section{Example of prestretch}

We consider a case of finite longitudinal strain applied to a straight, uniform, and $\ell$-long beam. The superimposed wave on this finite transformation is studied in detail.

\subsection{Static prestretch}

The arbitrary large pre-strain is obtained by imposing a static load $\mathrm{N}=F \mathbf{e}_{3}$ at the two ends of a finite beam (Fig. 2). If $F>0$ the beam undergoes a traction, and a compression if $F<0$. Here we consider a dead load, this induces that $\Delta \mathbf{q}$ and $\Delta \mathbf{m}$ vanish at the ends. The more interesting case of a follower load may introduce some specific features that would be analyzed in a dedicated paper.

For this simple case, the axial transformation is uniform:

$$
\mathbf{O M}=S(1+\epsilon) \mathbf{e}_{3}+\xi_{\alpha} \mathbf{e}_{\alpha}
$$



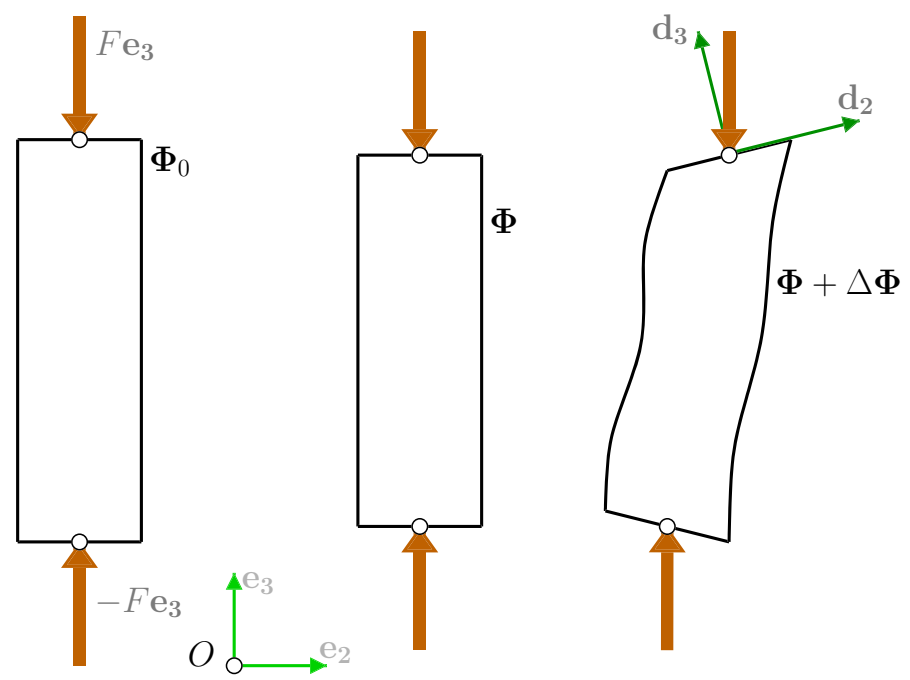

Fig. 2 Finite longitudinal pre-stress and additional vibration for an axial dead load. Left reference configuration, middle actual configuration, right actual configuration with additional vibrations. The sketch is provided in 2D for the sake of the clarity, and vibrations are presented with magnification

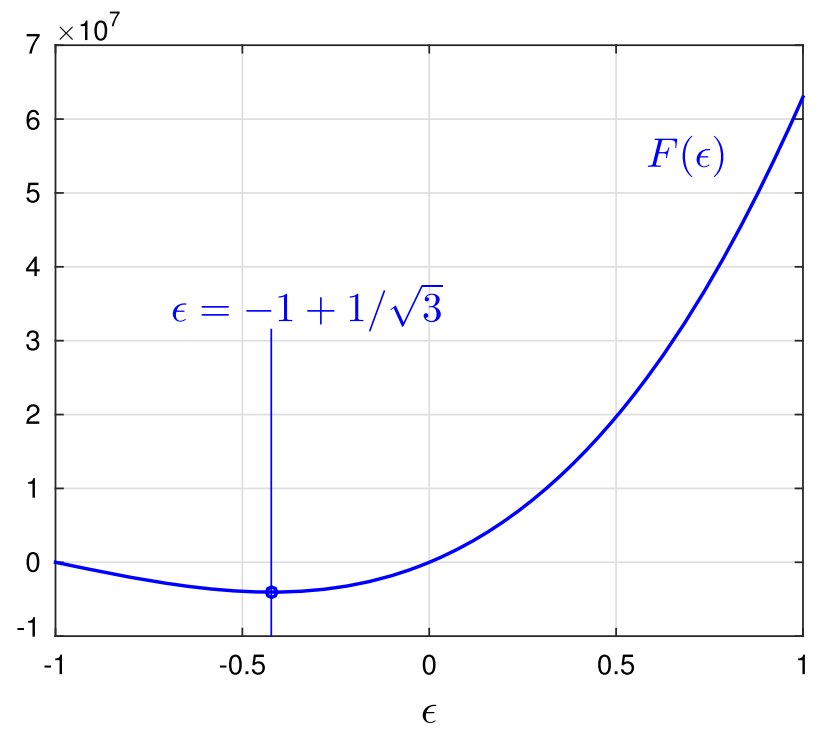

Fig. $3 F$ versus $\epsilon$. Computation is performed for a $\lambda+2 \mu=210 \mathrm{GPa}, A=1 \mathrm{~cm}^{2}$. Vertical units are Newtons (N)

where $\epsilon=\varepsilon_{3}$. The beam section does not undergo finite rotation: $\mathbf{d}_{\mathbf{i}}=\mathbf{D}_{\mathbf{i}}=\mathbf{e}_{\mathbf{i}}$ and $\boldsymbol{\kappa}=0$.

A general formulation of the force and moment acting on the beam section according to the total strain is given in the "Appendix." However, for the present load, direct integration can be performed. Using Eq. (16), $E_{33}=\epsilon(1+\epsilon / 2)$ is the only non-null component of the Green-Lagrange strain tensor. With Eq. (44), we directly have $\mathbf{F}=\operatorname{diag}(1,1,1+\epsilon)$ and then the nominal Piola-Kirchhoff stress, $\mathbf{P}=\mathbf{F S}$ by using the expression of $\mathbf{F}$ provided by Eq. (43). By direct integration along the beam section, we obtain $\mathrm{N}=\mathrm{Fe}_{\mathbf{3}}$ and then the nonlinear relation between the axial strain and the load:

$$
F=A(\lambda+2 \mu) \epsilon(1+\epsilon)\left(1+\frac{\epsilon}{2}\right) .
$$

This relation is presented in Fig. 3. The relation is monotonic for $-1+\frac{1}{\sqrt{3}} \leq \epsilon$ that gives a lower bound for the admissible strain. This bound is intrinsically related to the particular strain energy used for this example.

The problem of vibration superimposed on this pre-strain can be parametrized either by $F$ or by $\epsilon$. In view of the global philosophy of the calculation performed in the previous section, it is easier to use $\epsilon$ as a 
(non-dimensional) parameter. It is still possible to change the parametrization as the function $F(\epsilon)$ is bijective in the strain domain.

\subsection{Problem statement}

The first step is to compute Eqs. (45) and (46). Straightforward calculus gives, respectively:

$$
\begin{aligned}
\int_{\mathcal{B}} \mathbf{S}:\left(\Delta \mathbf{F}^{T} \delta \mathbf{F}\right) \mathrm{d} V= & \epsilon\left(1+\frac{\epsilon}{2}\right)\left((\lambda+2 \mu) \int_{\mathcal{C}} A \frac{\partial \Delta \varphi}{\partial S} \cdot \frac{\partial \delta \varphi}{\partial S}+\left(\mathbb{I} \frac{\partial \Delta \omega}{\partial S}\right) \cdot \frac{\partial \delta \boldsymbol{\omega}}{\partial S} \mathrm{~d} S\right. \\
& \left.+\lambda A \int_{\mathcal{C}} \Delta \boldsymbol{\omega} \cdot \delta \boldsymbol{\omega}+\Delta \omega_{3} \delta \omega_{3} \mathrm{~d} S\right)
\end{aligned}
$$

and

$$
\begin{aligned}
\int_{\mathcal{B}} \delta \mathbf{E}: \mathbf{C}(\Delta \mathbf{E}) \mathrm{d} V= & \int_{\mathcal{C}} \mu I_{3} \frac{\partial \Delta \omega_{3}}{\partial S} \frac{\partial \delta \omega_{3}}{\partial S}+(\lambda+2 \mu)(1+\epsilon)^{2} \sum_{\alpha=1}^{2} \mathbb{I}_{\alpha} \frac{\partial \Delta \omega_{\alpha}}{\partial S} \frac{\partial \delta \omega_{\alpha}}{\partial S} \mathrm{~d} S \\
& +\mu A \int_{\mathcal{C}}\left(\frac{\partial \Delta \varphi_{1}}{\partial S}-(1+\epsilon) \Delta \omega_{2}\right)\left(\frac{\partial \delta \varphi_{1}}{\partial S}-(1+\epsilon) \delta \omega_{2}\right) \mathrm{d} S \\
& +\mu A \int_{\mathcal{C}}\left(\frac{\partial \Delta \varphi_{2}}{\partial S}+(1+\epsilon) \Delta \omega_{1}\right)\left(\frac{\partial \delta \varphi_{2}}{\partial S}+(1+\epsilon) \delta \omega_{1}\right) \mathrm{d} S \\
& +(\lambda+2 \mu) A(1+\epsilon)^{2} \int_{\mathcal{C}} \frac{\partial \Delta \varphi_{3}}{\partial S} \frac{\partial \delta \varphi_{3}}{\partial S} \mathrm{~d} S .
\end{aligned}
$$

As expected, the geometrical contribution is at least linear in terms of $\epsilon$, whereas the material contribution is nonzero even for $\epsilon=0$. The integration by parts of the two contributions gives:

$$
\begin{aligned}
\int_{\mathcal{B}} \mathbf{S}:\left(\Delta \mathbf{F}^{T} \delta \mathbf{F}\right) \mathrm{d} V= & \epsilon\left(1+\frac{\epsilon}{2}\right)\left((\lambda+2 \mu)\left[A \frac{\partial \Delta \boldsymbol{\varphi}}{\partial S} \cdot \delta \boldsymbol{\varphi}+\left(\mathbb{I} \frac{\partial \Delta \boldsymbol{\omega}}{\partial S}\right) \cdot \delta \boldsymbol{\omega}\right]_{0}^{\ell} \ldots\right. \\
& -\int_{\mathcal{C}}(\lambda+2 \mu)\left(A \frac{\partial^{2} \Delta \varphi}{\partial S^{2}} \cdot \delta \boldsymbol{\varphi}+\left(\mathbb{I} \frac{\partial^{2} \Delta \boldsymbol{\omega}}{\partial S^{2}}\right) \cdot \delta \boldsymbol{\omega}\right) \\
& \left.-\lambda A\left(\Delta \boldsymbol{\omega} \cdot \delta \boldsymbol{\omega}+\Delta \omega_{3} \delta \omega_{3}\right) \mathrm{d} S\right), \\
\int_{\mathcal{B}} \delta \mathbf{E}: \mathbf{C}(\Delta \mathbf{E}) \mathrm{d} V= & -\int_{\mathcal{C}} \mu A\left(\left(\frac{\partial^{2} \Delta \varphi_{1}}{\partial S^{2}}-(1+\epsilon) \frac{\partial \Delta \omega_{2}}{\partial S}\right) \delta \varphi_{1}+\left(\frac{\partial^{2} \Delta \varphi_{2}}{\partial S^{2}}+(1+\epsilon) \frac{\partial \Delta \omega_{1}}{\partial S}\right) \delta \varphi_{2}\right. \\
& \left.+(1+\epsilon)\left(\left(\frac{\partial \Delta \varphi_{1}}{\partial S}-(1+\epsilon) \Delta \omega_{2}\right) \delta \omega_{2}-\left(\frac{\partial \Delta \varphi_{2}}{\partial S}+(1+\epsilon) \Delta \omega_{1}\right) \delta \omega_{1}\right)\right) \\
& +(\lambda+2 \mu)(1+\epsilon)^{2}\left(A \frac{\partial^{2} \Delta \varphi_{3}}{\partial S^{2}} \delta \varphi_{3}+\sum_{\alpha=1}^{2} \mathbb{I}_{\alpha} \frac{\partial^{2} \Delta \omega_{\alpha}}{\partial S^{2}} \delta \omega_{\alpha}\right)+\mu I_{3} \frac{\partial^{2} \Delta \omega_{3}}{\partial S^{2}} \delta \omega_{3} \mathrm{~d} S \\
& +\left[(\lambda+2 \mu) A(1+\epsilon)^{2}\left(\frac{\partial \Delta \varphi_{3}}{\partial S} \delta \varphi_{3}+\sum_{\alpha=1}^{2} \mathbb{I}_{\alpha} \frac{\partial \Delta \omega_{\alpha}}{\partial S} \delta \omega_{\alpha}\right)+\mu I_{3} \frac{\partial \Delta \omega_{3}}{\partial S} \delta \omega_{3}\right. \\
& \left.+\mu A\left(\left(\frac{\partial \Delta \varphi_{1}}{\partial S}-(1+\epsilon) \Delta \omega_{2}\right) \delta \varphi_{1}+\left(\frac{\partial \Delta \varphi_{2}}{\partial S}+(1+\epsilon) \Delta \omega_{1}\right) \delta \varphi_{2}\right)\right]_{0}^{\ell} .
\end{aligned}
$$

Combining geometrical and material contributions and inserting inertia, we obtain after rearrangement the weak form of the full vibrational problem (corresponding to Eq. (41) for this practical example):

$$
\begin{aligned}
\int_{0}^{\ell} & \left(\widetilde{G} A \frac{\partial^{2} \Delta \varphi_{1}}{\partial S^{2}}-\widehat{G} A \frac{\partial \Delta \omega_{2}}{\partial S}-\rho A \frac{\partial^{2} \Delta \varphi_{1}}{\partial t^{2}}\right) \delta \varphi_{1} \\
& +\left(\widetilde{E} I_{2} \frac{\partial^{2} \Delta \omega_{2}}{\partial S^{2}}+\widehat{G} A \frac{\partial \Delta \varphi_{1}}{\partial S}-\widetilde{G} A \Delta \omega_{2}-\rho I_{2} \frac{\partial^{2} \Delta \omega_{2}}{\partial t^{2}}\right) \delta \omega_{2}
\end{aligned}
$$




$$
\begin{aligned}
& +\left(\widetilde{G} A \frac{\partial^{2} \Delta \varphi_{2}}{\partial S^{2}}+\widehat{G} A \frac{\partial \Delta \omega_{1}}{\partial S}-\rho A \frac{\partial^{2} \Delta \varphi_{2}}{\partial t^{2}}\right) \delta \varphi_{2} \\
& +\left(\widetilde{E} I_{1} \frac{\partial^{2} \Delta \omega_{1}}{\partial S^{2}}-\widehat{G} A \frac{\partial \Delta \varphi_{2}}{\partial S}-\widetilde{G} A \Delta \omega_{1}-\rho I_{1} \frac{\partial^{2} \Delta \omega_{1}}{\partial t^{2}}\right) \delta \omega_{1} \\
& +\left(\widetilde{E} A \frac{\partial^{2} \Delta \varphi_{3}}{\partial S^{2}}-\rho A \frac{\partial^{2} \Delta \varphi_{3}}{\partial t^{2}}\right) \delta \varphi_{3}+\left(\widetilde{G} I_{3} \frac{\partial^{2} \Delta \omega_{3}}{\partial S^{2}}-2 \widetilde{D} A \Delta \omega_{3}-\rho I_{3} \frac{\partial^{2} \Delta \omega_{3}}{\partial t^{2}}\right) \delta \omega_{3} \mathrm{~d} S \\
& =\left[A\left(\widetilde{G} \frac{\partial \Delta \varphi_{1}}{\partial S}-\widehat{G} \Delta \omega_{2}\right) \delta \varphi_{1}+I_{2} \widetilde{E} \frac{\partial \Delta \omega_{2}}{\partial S} \delta \omega_{2}\right. \\
& \left.+A\left(\widetilde{G} \frac{\partial \Delta \varphi_{2}}{\partial S}+\widehat{G} \Delta \omega_{1}\right) \delta \varphi_{2}+I_{1} \widetilde{E} \frac{\partial \Delta \omega_{1}}{\partial S} \delta \omega_{1}+A \widetilde{E} \frac{\partial \Delta \varphi_{3}}{\partial S} \delta \varphi_{3}+I_{3} \widetilde{G} \frac{\partial \Delta \omega_{3}}{\partial S} \delta \omega_{3}\right]_{0}^{\ell}
\end{aligned}
$$

where we have introduced the effective rigidity moduli with respect to the finite strain:

$$
\widetilde{E}=(\lambda+2 \mu)\left(1+3 \epsilon\left(1+\frac{\epsilon}{2}\right)\right), \quad \widetilde{G}=\mu\left(1+K_{\nu} \epsilon\left(1+\frac{\epsilon}{2}\right)\right), \quad \widehat{G}=\mu(1+\epsilon), \quad \widetilde{D}=\lambda \epsilon\left(1+\frac{\epsilon}{2}\right)
$$

with a bulk-shear ratio

$$
K_{v}=\frac{\lambda+2 \mu}{\mu}=\frac{2(1+v)}{1-2 v}, \quad\left(K_{v}=3 \text { if } v=\frac{1}{8}\right) .
$$

Remark that $K_{v}$ has a lower bound for $v=0$, but no upper bound $\left(2 \leq K_{v}\right)$.

We observe that the full problem casts into two independent bending problems $\left(b_{1}\right)$ and $\left(b_{2}\right)$, a longitudinal $(l)$ and a torsional one $(t)$. Their strong formulation, essential, and natural boundary conditions are:

$$
\begin{aligned}
& \left(b_{1}\right) \text { bending in }\left(\mathbf{d}_{\mathbf{1}}, \mathbf{d}_{\mathbf{3}}\right) \text {-plane } \quad \widetilde{G} A \frac{\partial^{2} \Delta \varphi_{1}}{\partial S^{2}}-\widehat{G} A \frac{\partial \Delta \omega_{2}}{\partial S}=\rho A \frac{\partial^{2} \Delta \varphi_{1}}{\partial t^{2}} \\
& \widetilde{E} I_{2} \frac{\partial^{2} \Delta \omega_{2}}{\partial S^{2}}+\widehat{G} A \frac{\partial \Delta \varphi_{1}}{\partial S}-\widetilde{G} A \Delta \omega_{2}=\rho I_{2} \frac{\partial^{2} \Delta \omega_{2}}{\partial t^{2}} \\
& \text { natural }\left\{\begin{array}{c|c}
\widetilde{G} A \frac{\partial \Delta \varphi_{1}}{\partial \widehat{S}}-\widehat{G} A \Delta \omega_{2}=0 & \delta \varphi_{1}=0 \\
E I_{2} \frac{\partial \Delta \omega_{2}}{\partial S}=0 & \delta \omega_{2}=0
\end{array}\right\} \quad \text { essential, }
\end{aligned}
$$

$\left(b_{2}\right)$ bending in $\left(\mathbf{d}_{2}, \mathbf{d}_{3}\right)$-plane $\quad \widetilde{G} A \frac{\partial^{2} \Delta \varphi_{2}}{\partial S^{2}}+\widehat{G} A \frac{\partial \Delta \omega_{1}}{\partial S}=\rho A \frac{\partial^{2} \Delta \varphi_{2}}{\partial t^{2}}$

$$
\begin{gathered}
\widetilde{E} I_{1} \frac{\partial^{2} \Delta \omega_{1}}{\partial S^{2}}-\widehat{G} A \frac{\partial \Delta \varphi_{2}}{\partial S}-\widetilde{G} A \Delta \omega_{1}=\rho I_{1} \frac{\partial^{2} \Delta \omega_{1}}{\partial t^{2}} \\
\text { natural }\left\{\begin{array}{c|c}
\widetilde{G} A \frac{\partial \Delta \varphi_{2}}{\partial \stackrel{S}{\overparen{G}} A \Delta \omega_{1}=0} & \delta \varphi_{2}=0 \\
\widetilde{E} I_{1} \frac{\partial \Delta \omega_{1}}{\partial S}=0 & \delta \omega_{1}=0
\end{array}\right\} \quad \text { essential, }
\end{gathered}
$$

(l) longitudinal vibration along $\mathbf{d}_{\mathbf{3}} \quad \widetilde{E} A \frac{\partial^{2} \Delta \varphi_{3}}{\partial S^{2}}=\rho A \frac{\partial^{2} \Delta \varphi_{3}}{\partial t^{2}}$ natural $\widetilde{E} A \frac{\partial \Delta \varphi_{3}}{\partial S}=0 \mid \delta \varphi_{3}=0$ essential,

( $t$ ) torsional vibration around $\mathbf{d}_{3} \quad \widetilde{G} I_{3} \frac{\partial^{2} \Delta \omega_{3}}{\partial S^{2}}-2 \widetilde{D} A \Delta \omega_{3}=\rho I_{3} \frac{\partial^{2} \Delta \omega_{3}}{\partial t^{2}}$ natural $\widetilde{G} I_{3} \frac{\partial \Delta \omega_{3}}{\partial S}=0 \mid \delta \omega_{3}=0$ essential,

\subsection{Brief analysis}

Even for this practical example, the result is very rich. We present here some particular points that must be highlighted. We restrict ourselves to a brief analysis as each of these points may motivate a deep analysis being beyond the general objective of this paper. 


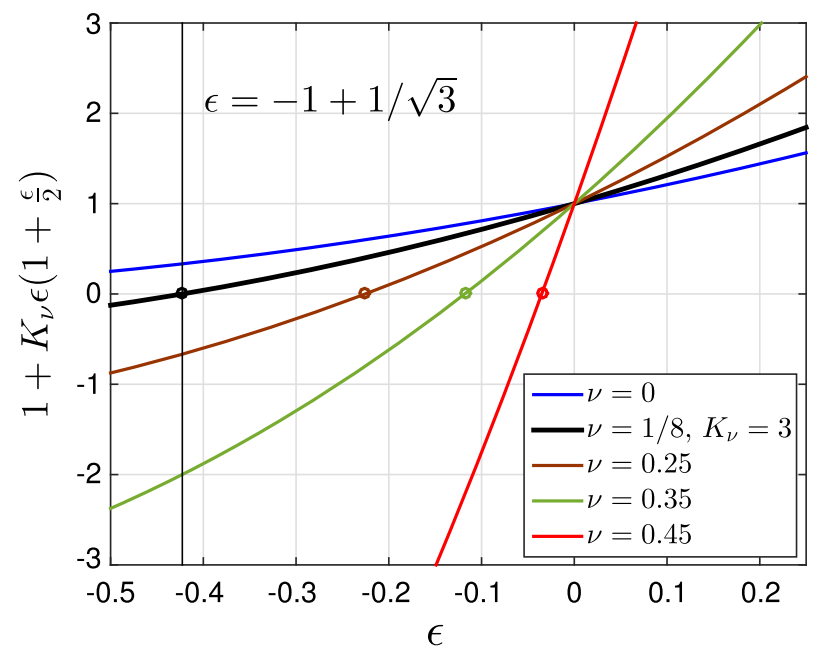

Fig. 4 Analysis of the effective modulus factor $1+3 \epsilon(1+\epsilon / 2)$ (black) and $1+K_{\nu} \epsilon(1+\epsilon / 2)$ (color) with respect to the finite strain $\epsilon$ and for various Poisson ratios $v$. Dots denotes the canceling of these factors

\subsubsection{Instability}

The sensitivity of the effective moduli versus the finite strain is illustrated on Fig. 4. These moduli are both sensitive to finite strain and the Poisson's ratio of the material.

At this step, we have to draw our attention to the fact that some effective moduli may be zero for some critical finite longitudinal compression. This is the case for $\widetilde{E}$ if $\epsilon=-1+1 / \sqrt{3}$ (i.e., for $\frac{\partial F}{\partial \epsilon}=0$ ). For this critical strain, the natural boundary conditions are no more able to fix the slope of the longitudinal vibration $\left(\frac{\partial \Delta \varphi_{3}}{\partial S}\right)$ and of the transverse rotation $\left(\frac{\partial \Delta \omega_{1}}{\partial S}\right.$ and $\left.\frac{\partial \Delta \omega_{2}}{\partial S}\right)$. In fact the longitudinal force $\widetilde{E} A \frac{\partial \Delta \varphi_{3}}{\partial S}$ and the bending moment $\left(\widetilde{E} I_{1} \frac{\partial \Delta \omega_{1}}{\partial S}\right.$ and $\left.\widetilde{E} I_{2} \frac{\partial \Delta \omega_{2}}{\partial S}\right)$ at the boundaries are zero (as expected for natural boundary condition), but the constraint on the kinematical quantities is no more effective. This may be interpreted as a particular instability of the structure. Of course, this effect is obtained for a limit value of the strain.

More interesting, this global phenomenon is observed for $\widetilde{G}$ for $\epsilon \geq-1+1 / \sqrt{3}$ and $v \geq 1 / 8$. This induces possible instabilities, attainable even for moderate compression, for torsional wave and transverse displacement $\left(\frac{\partial \Delta \varphi_{1}}{\partial S}\right.$ and $\frac{\partial \Delta \varphi_{2}}{\partial S}$ ) with zero angle $\Delta \omega_{1}$ and $\Delta \omega_{2}$ at the ends. Again, all these instabilities are concomitant in the sense that they arise at the same compression value.

In fact for such zero moduli, the whole problem must be rewritten. In particular, the definition of the boundary conditions must be readdressed in order to clearly consider various loadings (dead load or slipping load for example). Even if these phenomena appear for particularly large compression, these observations look to open new perspectives on possible instabilities at the ends of the compressed elongated structures.

\subsubsection{Shear force and gliding angle}

For the bending problem ${ }_{2}$ the first natural boundary conditions are related to the absence of the transverse force. However, because $\widetilde{G} \neq \widehat{G}$ for $\epsilon \neq 0$, we observe that these boundary conditions induce a non-vanishing gliding angle $\left(\gamma_{1}=\frac{\partial \Delta \varphi_{2}}{\partial S}+\Delta \omega_{1}\right.$ and $\left.\gamma_{2}=\frac{\partial \Delta \varphi_{1}}{\partial S}-\Delta \omega_{2}\right)$ at the ends for a nonzero pre-stress.

\subsubsection{Buckling}

For the bending problem, buckling is related to critical finite compressive strain for which the stiffness operator admits some non-trivial static solutions. Let us consider $\left(b_{1}\right)$ for example and impose harmonic displacement $\Delta \varphi_{1}(S)=\Phi_{1} \exp (i k S)$ and angle $\Delta \omega_{2}(S)=\Omega_{2} \exp (i k S)$, then the stiffness matrix becomes:

$$
\mathbb{K}=\left(\begin{array}{cc}
-k^{2} \widetilde{G} A & -i k \widehat{G} A \\
i k \widehat{G} A & -k^{2} \widetilde{E} I_{2}-\widetilde{G} A
\end{array}\right)
$$




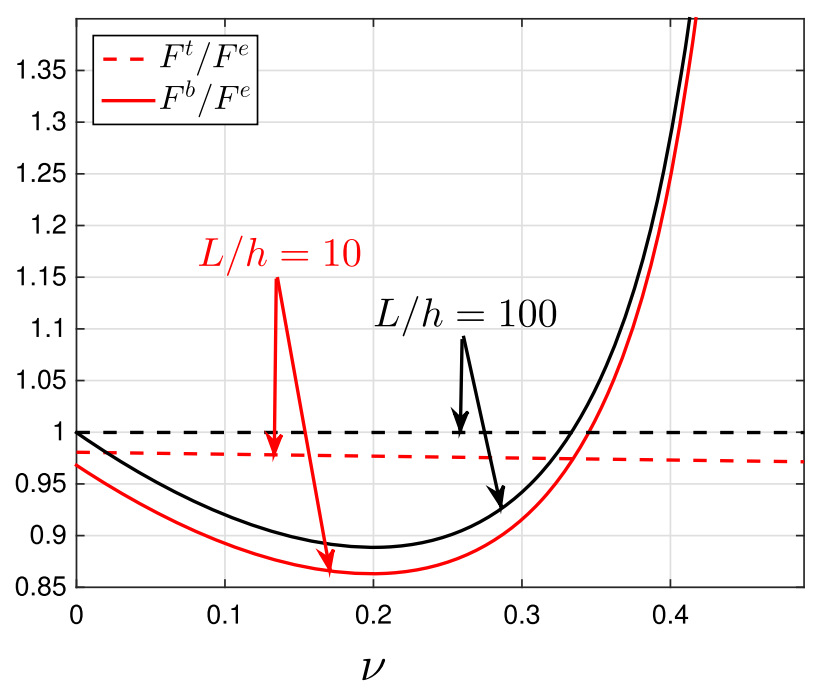

Fig. 5 Non-dimensional buckling load proposed in this paper $F^{b} / F^{e}$ (line) and from Timoshenko and Gere $F^{t} / F^{e}(d o t s)$, where $F^{e}$ is the Euler prediction. These loads are presented with respect to $v$ and for thick beam $(r e d, \ell / h=10)$ and thin beam (black, $\ell / h=100$ ), for higher slenderness ratio the curves are mainly unchanged. Simulation is performed for pinned boundary conditions $(k=\pi / \ell)$ and $h \times h$ square cross section $\left(\kappa^{t}=5 / 6\right)$

and a non-trivial solution appears for $\operatorname{det}(\mathbb{K})=0$, i.e., $\left.A k^{2}\left(A\left(\widetilde{G}^{2}-\widehat{G}^{2}\right)+k^{2} \widetilde{E} \widetilde{G} I_{2} k^{2}\right)\right)=0$, this corresponds to a non-trivial wavenumber $k$ satisfying

$$
\widetilde{G} \widetilde{E} I_{2} k^{2}=A\left(\widehat{G}^{2}-\widetilde{G}^{2}\right) .
$$

Here $k$ is imposed by boundary condition, and the later equation can be solved to define the critical strain $\epsilon$ inducing buckling. In a second step, the buckling load $F^{b}$ can be computed according to Eq. (47). This estimation can be compared with the classical Euler buckling load $F^{e}=-E I_{2} k^{2}$ or with the prediction from Timoshenko and Gere [8,39]

$$
F^{t}=-\frac{E I_{2} k^{2}}{1+\frac{E I_{2}}{G A} k^{2}}
$$

where $E$ is Young's modulus and $G=\kappa^{t} \mu$ is the corrected shear modulus ( $\kappa^{t}=5 / 6$ for rectangular cross section).

Simulation is performed for $F^{b}, F^{e}$, and $F^{t}$, for a straight beam of length $\ell$ and $h \times h$ cross section with pinned boundary conditions $(k=\pi / \ell)$. The non-dimensional ratio $F^{b} / F^{e}$ and $F^{t} / F^{e}$ depends on the Poisson's ratio and the slenderness ratio $\ell / h$. In Fig. 5 the behavior of $F^{b} / F^{e}$ and $F^{t} / F^{e}$ is presented versus $\nu$, for thick and thin beams $(\ell / h=10$ and $\ell / h=100$, respectively). The Timoshenko hypothesis predicts mainly linear dependency to the Poisson's ratio, whereas the model presented here gives more complex results. The Timoshenko model supposes that Euler buckling load is always overestimated $\left(F^{t} / F^{e} \leq 1\right)$. For the model presented in this paper, the Euler prediction is an overestimation only for moderate Poisson's ratio $(v \lesssim 1 / 3)$ and an underestimation for higher Poisson's ratio $(v \gtrsim 1 / 3)$. Last the present model suggests an infinite load for incompressible material. Our model and the Timoshenko model propose the same estimation for $v \sim 0$ and $v \sim 1 / 3$ whatever the $\ell / h$. On the one hand, the rigid section hypothesis used in the present model is consistent with $v \sim 0$ and corresponds to Timoshenko hypothesis. On the other hand, this may be seen as a partial validation as the Timoshenko prediction is considered efficient for steel or aluminum-like material $(v \sim 1 / 3)$.

\subsubsection{String model as an asymptotic}

The string model can be obtained by asymptotic analysis. We illustrate this assertion by considering the problem $\left(b_{1}\right)$. 
becomes

One way is to consider that $\mu /(\lambda+2 \mu) \rightarrow 0$ (large Poisson's ratio), in such a case the first line of $\left(b_{1}\right)$

$$
(\lambda+2 \mu) \epsilon\left(1+\frac{\epsilon}{2}\right) A \frac{\partial^{2} \Delta \varphi_{1}}{\partial S^{2}}=\rho A \frac{\partial^{2} \Delta \varphi_{1}}{\partial t^{2}} .
$$

Another way is to consider a very thin beam $\sqrt{I_{2}} \ll A$. In such a case the second line of $\left(b_{1}\right)$ becomes:

$$
\widehat{G} A \frac{\partial \Delta \varphi_{1}}{\partial S}-\widetilde{G} A \Delta \omega_{2}=0 ;
$$

inserting this relation in the first line of $\left(b_{1}\right)$ leads to:

$$
\frac{\widetilde{G}^{2}-\widehat{G}^{2}}{\widetilde{G}} A \frac{\partial^{2} \Delta \varphi_{1}}{\partial S^{2}}=\rho A \frac{\partial^{2} \Delta \varphi_{1}}{\partial t^{2}}
$$

where $\frac{\widetilde{G}^{2}-\widehat{G}^{2}}{\widetilde{G}} \simeq \epsilon(2 \lambda+2 \mu)$.

The last way is to impose a kinematical constraint: the absence of gliding angle $\gamma_{2}$. In such a case the first line of $\left(b_{1}\right)$ becomes

$$
(\widetilde{G}-\widehat{G}) A \frac{\partial^{2} \Delta \varphi_{1}}{\partial S^{2}}=\rho A \frac{\partial^{2} \Delta \varphi_{1}}{\partial t^{2}}
$$

where $\widetilde{G}-\widehat{G} \simeq(\lambda+\mu) \epsilon$.

Considering in first approximation $F=(\lambda+2 \mu) \epsilon A$, we obtain for the three hypotheses:

$$
\widetilde{F} \frac{\partial^{2} \Delta \varphi_{1}}{\partial S^{2}}=\rho A \frac{\partial^{2} \Delta \varphi_{1}}{\partial t^{2}}
$$

where $\widetilde{F}=F$ for the first hypothesis, $\widetilde{F}=\frac{2(\lambda+\mu)}{\lambda+2 \mu} F=F /(1-v)$ for the second, and $\widetilde{F}=F /(2(1-v))$ for the third.

These ways to recover the string equation from general theory on vibration superimposed on large pre-stress need of course some further investigations. In particular, it must be noticed that these three hypotheses are distinct as they are related to material properties, geometrical properties, and kinematics, respectively. Hence, it is highly possible that more than one hypothesis can be observed in a specific example (that induces finest analysis). This suggests than there is more than one intermediate problem between the simple string model and the vibration of a beam supporting finite elongation.

In summary, the present approach includes string and ropes models for some asymptotical case what looks to confirm the efficiency of the proposed development (for example, this remarkable result is not present in the Haringx'model). As the present model is valid even for non-asymptotical situation, it looks to be pertinent to establish some intermediate problem. This work may extend other approaches dedicated to the string of musical instruments $[23,40]$.

\section{Concluding remarks}

The construction of a nonlinear beam model may be performed in two very distinct ways. The first focuses on the dynamics of a curvilinear segment supporting mechanical significations (Cosserat-like structure in our case) [41]. In the second way, a three-dimensional slender rod-like body is the primal object and eventual kinematical constraints are imposed. Last, reduction to a one-dimensional element is performed by asymptotic analysis or integration over the cross section. This latter is the methodology used all along the present paper, and in that sense the models proposed here for (i) finite transformation and (ii) superimposed waves are both induced geometrically exact models (using Antman's terminology [42]). Strictly speaking, we do not analyze the superimposed waves upon a finite transformation of a beam, but we are modeling in terms of the beam's vibrations the superimposed perturbations upon a three-dimensional body supporting finite transformation.

This methodology avoids some counterpart that may be induced by freezing the internal kinematics during integration along the cross section. For example, it permits a rigorous analysis of the perturbed external virtual power (Sect. 5.2) that may not be carried out if the perturbations were performed on a frozen beam element. 
An other advantage of the methodology is that it leads to a self-adjoint operator for internal virtual power, and hence superimposed waves play a similar role as the virtual transformation. The decomposition of the internal energy into geometrical and material contribution seems to be new from our knowledge.

All along the paper, the number of hypotheses is reduced to a strict minimum: (i) a slender body, (ii) the transformation is compatible (no contact or penetration along the transformation), (iii) no viscous effect (the strain rate is not introduced), (iv) the cross section is rigid. Hence, this work may be exploited for a body with varying cross section, for heterogeneous and anisotropic medium, and for any constitutive relation. However, a source of confusion must be clarified: Even if the elasticity tensor is not used during the finite transformation, we have introduced an effective elasticity tensor $\mathrm{C}$ during the analysis of the superimposed vibration (Eq. (38)). This tensor is not necessarily related to a free energy and must be seen as a tangent elasticity tensor around the prescribed finite transformation. However, we do not consider additional plasticity induced by wave propagation. Concerning the time dependence, the model for superimposed waves is still valid if the finite strain of the body is related to a larger time scale than the vibration's timescale, or if the body supports a fast rigid motion [18,19,22]. This holds true for the external loads, that may change but at low corotational rate (with respect to wave motion).

Even if the general solution for the weak formulation of the beam dynamics may look non explicit in the general case (Sect. 5.4), the example proposed in the next sections shows that this model easily supports analytical and explicit formulations for a given problem. The authors expect that this will help rigorous and explicit analysis of interesting phenomena. An example is presented in two steps. First, for Helmholtz free energy the force and moment acting on the cross section for any transformation are given (in the "Appendix"), and the density of virtual power is given explicitly. Second, for a finite longitudinal load, the full vibration equations are presented. This example highlights a large number of phenomena related to stability and buckling and gives some glimpse on the asymptotic link between string and pre-stretched beam. More generally, as induced geometrically exact model, the presented approach encompasses beams, rods, string, and ropes, in term of finite transformation and superimposed vibrations.

Among the possible extension of the approach, we can introduce a less constrained kinematical hypothesis, by allowing affine transformation of the section (i.e., reduction of size of the planar section) or warping [30]. Introduction of a visco-elastic constitutive law may be interesting, too. The explicit solution for other elastic potentials is another objective (Mooney-Rivlin for example). A deep analysis of the boundary conditions (in particular the effect of dead and followers boundary conditions) may have to be conducted, too [43]. In terms of buckling, this deformable model can be confronted to Beck or other models.

\section{Appendix: Force and moment}

Here, we give the expression of force $\mathrm{N}=N_{i} \mathbf{d}_{\mathbf{i}}$ and moment $\mathrm{M}=M_{i} \mathbf{d}_{\mathbf{i}}$ acting on a beam section in an unperturbed situation.

We begin with expression Eq. (33), where we see that the free energy has to be integrated over the beam section. Indeed, this free energy may be synthesized as follows:

$$
\psi=\frac{\lambda}{8}\left(2 Z_{3}+\|\mathbf{Z}\|^{2}\right)^{2}+\frac{\mu}{2}\left(Z_{1}^{2}+Z_{2}^{2}+\frac{1}{2}\left(2 Z_{3}+\|\mathbf{Z}\|^{2}\right)^{2}\right) .
$$

where

$$
\mathbf{Z}=Z_{i} \mathbf{d}_{\mathbf{i}}=\boldsymbol{\varepsilon}+\boldsymbol{\kappa} \times \mathbf{G M}=\frac{\partial \mathbf{O M}}{\partial S}-\mathbf{d}_{\mathbf{3}}
$$

By integrating this energy density over the cross section, we obtain an expression of the Timoshenko free energy per unit length, e.g., [35]:

$$
\Psi=\frac{\mu}{2} A \varepsilon_{1}^{2}+\frac{\mu}{2} A \varepsilon_{2}^{2}+\left(\frac{\lambda}{2}+\mu\right) A \varepsilon_{3}^{2}+\left(\frac{\lambda}{2}+\mu\right) I_{\alpha} \kappa_{\alpha}^{2}+\frac{\mu}{2} I_{3} \kappa_{3}^{2}+\Psi^{*}(\varepsilon, \kappa)
$$

in which $\Psi^{*}(\boldsymbol{\varepsilon}, \boldsymbol{\kappa})$ is a polynomial function of degree more than two (induced by $\mathbf{H}^{T} \mathbf{H}$ in the Green-Lagrange strain tensor Eq. (15)), $A$ is the area of the cross section. $I_{i}$ are components of the tensor of quadratic moment $\mathbb{I}$ of a (rigid) beam section, and Cosserat directors bases $\mathbf{d}_{\alpha}(S, t)$ are directed along the principal axes of inertia of the cross section. 
Going back to Eq. (17), the components of force and moment are:

$$
\begin{aligned}
N_{1} & =\int_{\mathcal{S}} \mu Z_{1}+(\lambda+2 \mu) Z_{1}\left(Z_{3}+\frac{1}{2}\|\mathbf{Z}\|^{2}\right) \mathrm{d} A, \\
N_{2} & =\int_{\mathcal{S}} \mu Z_{2}+(\lambda+2 \mu) Z_{2}\left(Z_{3}+\frac{1}{2}\|\mathbf{Z}\|^{2}\right) \mathrm{d} A, \\
N_{3} & =\int_{\mathcal{S}}(\lambda+2 \mu)\left(Z_{3}+\frac{1}{2}\|\mathbf{Z}\|^{2}+Z_{3}^{2}+\frac{1}{2}\|\mathbf{Z}\|^{2} Z_{3}\right) \mathrm{d} A, \\
M_{1} & =\int_{\mathcal{S}}(\lambda+2 \mu) \xi_{2}\left(Z_{3}+\frac{1}{2}\|\mathbf{Z}\|^{2}+Z_{3}^{2}+\frac{1}{2}\|\mathbf{Z}\|^{2} Z_{3}\right) \mathrm{d} A, \\
M_{2} & =-\int_{\mathcal{S}}(\lambda+2 \mu) \xi_{1}\left(Z_{3}+\frac{1}{2}\|\mathbf{Z}\|^{2}+Z_{3}^{2}+\frac{1}{2}\|\mathbf{Z}\|^{2} Z_{3}\right) \mathrm{d} A, \\
M_{3} & =\int_{\mathcal{S}}\left(\xi_{1} Z_{2}-\xi_{2} Z_{1}\right)\left(\mu+(\lambda+2 \mu)\left(Z_{3}+\frac{1}{2}\|\mathbf{Z}\|^{2}\right) \mathrm{d} A .\right.
\end{aligned}
$$

Explicit integration is performed only for the leading term and gives:

$$
\begin{aligned}
& N_{1}=\mu \varepsilon_{1}+(\lambda+2 \mu)\left(A \varepsilon_{1} \varepsilon_{3}-I_{1} \kappa_{1} \kappa_{3}\right)+\frac{\lambda+2 \mu}{2} \int_{\mathcal{S}} Z_{1}\|\mathbf{Z}\|^{2} \mathrm{~d} A, \\
& N_{2}=\mu \varepsilon_{2}+(\lambda+2 \mu)\left(A \varepsilon_{2} \varepsilon_{3}-\mathbb{I}_{22} \kappa_{2} \kappa_{3}\right)+\frac{\lambda+2 \mu}{2} \int_{\mathcal{S}} Z_{2}\|\mathbf{Z}\|^{2} \mathrm{~d} A, \\
& N_{3}=(\lambda+2 \mu)\left(A \varepsilon_{3}+A \varepsilon_{3}^{2}+I_{1} \kappa_{1}^{2}+\mathbb{I}_{22} \kappa_{2}^{2}+\frac{1}{2} \sum_{i}\left(A \varepsilon_{i}^{2}+I_{i i} \kappa_{i}^{2}\right)+\frac{1}{2} \int_{\mathcal{S}}\|\mathbf{Z}\|^{2} Z_{3} \mathrm{~d} A\right), \\
& M_{1}=(\lambda+2 \mu)\left(I_{1} \kappa_{1}+\int_{\mathcal{S}} \xi_{2}\left(\frac{1}{2}\|\mathbf{Z}\|^{2}+Z_{3}^{2}+\frac{1}{2}\|\mathbf{Z}\|^{2} Z_{3}\right) \mathrm{d} A\right), \\
& M_{2}=(\lambda+2 \mu)\left(\mathbb{I}_{22} \kappa_{2}-\int_{\mathcal{S}} \xi_{1}\left(\frac{1}{2}\|\mathbf{Z}\|^{2}+Z_{3}^{2}+\frac{1}{2}\|\mathbf{Z}\|^{2} Z_{3}\right) \mathrm{d} A\right), \\
& M_{3}=\mu I_{3} \kappa_{3}+(\lambda+2 \mu) \int_{\mathcal{S}}\left(\xi_{1} Z_{2}-\xi_{2} Z_{1}\right)\left(Z_{3}+\frac{1}{2}\|\mathbf{Z}\|^{2}\right) \mathrm{d} A .
\end{aligned}
$$

The nonlinear term is always proportional to $\lambda+2 \mu$.

\section{References}

1. Villain, G., Abraham, O., Le Marrec, L., Rakotomanana, L.R.: Determination of the bulk elastic moduli of various concrete by resonance frequency analysis of slabs submitted to degradations. In: Proceedings 7 th International Symposium on Destructive Testing in Civil Engineering, pp. 881-886 (2009)

2. Etcheverry, J.I., Sánchez, G.A.: Resonance frequencies of parallelepipeds for determination of elastic moduli: an accurate numerical treatment. J. Sound Vib. 321, 631-646 (2009)

3. Forgit, C., Lemoine, B., Le Marrec, L., Rakotomanana, L.R.: A Timoshenko-like model for the study of three-dimensional vibrations of an elastic ring of general cross-section. Acta Mech. 227(9), 2543-2575 (2016)

4. Ajith, V., Gopalakrishnan, S.: Wave propagation in a porous composite beam: Porosity determination, location and quantification. Int. J. Solids Struct. 50(3), 556-569 (2013)

5. Puchegger, S., Loidl, D., Kromp, K., Peterlik, H., Weiss, R.: Extension of the resonant beam technique to highly anisotropic material. J. Sound Vib. 279, 1121-1129 (2005)

6. Aylor, W.R., Roland, E., Ploeg, H., Hertig, D., Klabunde, R., Warner, M.D., Hobatho, M.C., Rakotomanana, L., Clift, S.E.: Determination of orthotropic bone materials properties using FEA and modal analysis. J. Biomech. 35, 767-773 (2002)

7. Challamel, N., Rakotomanana, L., Le Marrec, L.: A dispersive wave equation using nonlocal elasticity. C. R. Mec. 337(8), 591-595 (2009)

8. Reddy, J.N.: Nonlocal theories for bending, buckling and vibration of beams. Int. J. Eng. Sci. 45(2), 288-307 (2007)

9. Eisenberger, M., Clastornik, J.: Vibrations and buckling of a beam on a variable Winkler elastic foundation. J. Sound Vib. 115(2), 233-241 (1987) 
10. Antman, S.S.: The equations for large vibrations of strings. Am. Math. Mon. 87(5), 359-370 (1980)

11. Han, S.M., Benaroya, H., Wei, T.: Dynamics of transversely vibrating beams using four engineering theories. J. Sound Vib. 225, 935-988 (1999)

12. Yoon, J., Ru, C.Q., Mioduchowski, A.: Timoshenko-beam effects on transverse wave propagation in carbon nanotubes. Compos. Part B 35, 87-93 (2004)

13. Hsu, J.C., Chang, R.P., Chang, W.J.: Resonance frequency of chiral single-walled carbon nanotubes using Timoshenko beam theory. Phys. Lett. A 372, 2757-2759 (2008)

14. Ekwaro-Osire, S., Maithripala, D.H.S., Berg, J.M.: A series expansion approach to interpreting the spectra of the Timoshenko beam. J. Sound Vib. 240, 667-678 (2001)

15. Bhaskar, A.: Elastic waves in Timoshenko beams: 'the lost and found' of an eigenmode. Proc. R. Soc. Lond. A Math. Phys. Sci. 465, 255-295 (2009)

16. Chan, K.T., Wang, X.Q., So, R.M.C., Reid, S.R.: Superposed standing waves in a Timoshenko beam. Proc. R. Soc. Lond. A Math. Phys. Sci. 458, 83-108 (2002)

17. Cosserat, E., Cosserat, F.: Théorie des Corps déformables. A. Hermann et Fils, Paris (1909)

18. Kang, B., Tan, C.A.: Elastic wave motions in an axially strained, infinitely long rotating Timoshenko shaft. J. Sound Vib. 213, 467-482 (1998)

19. Pierre, C., Ulsoy, A.G.: Consistent modeling of rotating Timoshenko shafts subject to axial loads. Ann. Arbor 1050, 48109$2125(1992)$

20. Kovacs, A.P.: The nonlinear vibration analysis of a clamped-clamped beam by a reduced multibody method. Nonlinear Dyn. 11, 121-141 (1996)

21. Larsen, J.W., Nielsen, S.R.K.: Non-linear dynamics of wind turbine wings. Int. J. Non-Linear Mech. 41(5), 629-643 (2006)

22. Chabassier, J., Duruflé, M.: Energy based simulation of a Timoshenko beam in non-forced rotation. Influence of the piano hammer shank flexibility on the sound. J. Sound Vib. 333(26), 7198-7215 (2014)

23. Chabassier, J., Imperiale, S.: Stability and dispersion analysis of improved time discretization for simply supported prestressed Timoshenko systems. Application to the stiff piano string. Wave Motion 50(3), 456-480 (2013)

24. Simo, J.C.: A finite strain beam formulation. Comput. Methods Appl. Mech. Eng. 49, 55-70 (1985)

25. Simo, J.C., Marsden, J.E., Krishnaprasad, P.S.: The Hamiltonian structure of nonlinear elasticity: the material and convective representations of solids, rods and plates. Arch. Ration. Mech. Anal. 104, 125-183 (1988)

26. Irschik, H., Gerstmayr, J.: A continuum mechanics based derivation of Reissners large-displacement finite-strain beam theory: the case of plane deformations of originally straight Bernoulli-Euler beams. Acta Mech. 206(1), 1-21 (2009)

27. Irschik, H., Gerstmayr, J.: A continuum-mechanics interpretation of Reissner's non-linear shear-deformable beam theory. Math. Comput. Model. Dyn. Syst. 17(1), 19-29 (2011)

28. Reissner, E.: On one-dimensional finite-strain beam theory: the plane problem. Z. Angew. Math. Phys. (ZAMP) 23(5), 795-804 (1972)

29. Simo, J.C., Vu-Quoc, L.: On the dynamics in space of rods undergoing large motions: a geometrically exact approach. Comput. Methods Appl. Mech. Eng. 66(2), 125-161 (1988)

30. Simo, J.C., Vu-Quoc, L.: A geometrically-exact rod model incorporating shear and torsion-warping deformation. Int. J. Solids Struct. 27(3), 371-393 (1991)

31. Lerbet, J.: Nonlinear dynamics of curvilinear systems. A new coordinate-free approach. J. Appl. Math. Mech. 69, 917-924 (2005)

32. Auciello, N.M., Ercolano, A.: A general solution for dynamic response of axially loaded non-uniform Timoshenko beams. Int. J. Solids Struct. 41, 4861-4874 (2004)

33. Gonzalez, O., Stuart, A.M.: A First Course in Continuum Mechanics. Cambridge University Press, Cambridge (2008)

34. Marsden, J.E., Hughes, T.J.R.: Mathematical Foundations of Elasticity. Prentice-Hall, Upper Saddle River (1983)

35. Rakotomanana, L.R.: Eléments de Dynamique des Solides et Structures Déformables. Presses Polytechniques et Universitaires Romandes, Collection Mécanique, Lausanne (2009)

36. Nakahara, M.: Geometry, Topology and Physics, Graduate Student Series in Physics. In: Brewer, D.F. (ed.). Institute of Physics Publishing, Bristol (1996)

37. Bideau, N., Eyi-Assoumou, P., Le Marrec L., Rakotomanana, L.: On the dynamics of shearable beams: general considerations and application to the vibrations of a non-linear Timoshenko beam. In: Ribeiro P., Amabili M. (eds.) Geometrically Nonlinear Vibrations of Structures, pp. 233-236 (2007)

38. Criesfield, M.A., Jelenić, G.: Objectivity of strain measures in the geometrically exact three-dimensional beam theory and its finite-element implementation. Proc. R. Soc. Lond. A Math. Phys. Sci. 455, 1125-1147 (1999)

39. Timoshenko, S.P., Gere, J.C.: Theory of Elastic Stability. McGraw Hill, New York (1961)

40. Watzky, A.: Non-linear three-dimensional large-amplitude damped free vibration of a stiff elastic stretched string. J. Sound Vib. 153(1), 125-142 (1992)

41. Antman, S.S.: Kirchhoffs problem for nonlinearly elastic rods. Q. Appl. Math. 32(3), 221-240 (1974)

42. Antman, S.S., Schuricht, F.: The critical role of the base curve for the qualitative behavior of shearable rods. Math. Mech. Solids 8, 75-102 (2003)

43. Antman, S.S., Lanza de Cristoforis, M.: Peculiar instabilities due to the clamping of shearable rods. Int. J. Non-Linear Mech. 32, 31-54 (1997) 